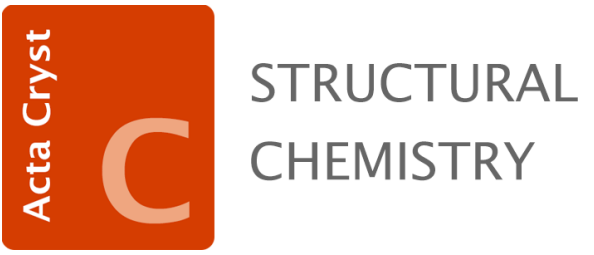

Volume 74 (2018)

Supporting information for article:

Structures and energetic properties of 4-halobenzamides

Aleksandra Piontek, Elwira Bisz, Błażej Dziuk, Roman Szostak and Michal Szostak 


\section{(4-Iodophenyl)(morpholino)methanone (1a)}

\section{Crystal data}

Chemical formula $\quad \mathrm{C}_{11} \mathrm{H}_{12} \mathrm{INO}_{2}$

$M_{\mathrm{r}} \quad 317.12$

Crystal system, space group Monoclinic, $P 2{ }_{1} / c$

Temperature (K) $\quad 100$

$\begin{array}{ll}a, b, c(\AA) & 10.5193(3), 12.2078(4), 8.8674(3) \\ \beta\left({ }^{\circ}\right) & 93.763(3) \\ V\left(\left(^{3}\right)\right. & 1136.27(6) \\ Z & 4 \\ \text { Radiation type } & \text { Mo Ka } \\ \mu\left(\mathrm{mm}^{-1}\right) & 2.80 \\ \text { Crystal size }(\mathrm{mm}) & 0.5 \times 0.4 \times 0.3\end{array}$

Data collection

Diffractometer Xcalibur

Absorption correction

No. of measured, independent and

observed $[I>2 \sigma(I)]$ reflections

$R_{\text {int }}$

$(\sin \theta / \lambda)_{\max }\left(\AA^{-1}\right)$

0.617

Refinement

$R\left[F^{2}>2 \sigma\left(F^{2}\right)\right], w R\left(F^{2}\right), S$

No. of reflections

No. of parameters

$\mathrm{H}$-atom treatment

$\Delta \rho_{\max }, \Delta \rho_{\min }\left(\mathrm{e} \AA^{-3}\right)$
$0.017,0.044,1.07$

2207

144

$\mathrm{H}$ atoms treated by a mixture of independent and constrained refinement

$0.42,-0.52$

Computer programs: CrysAlis CCD (Oxford Diffraction Ltd., 2008), SHELXS2014/7

(Sheldrick, 2014), SHELXL2014/7 (Sheldrick, 2014), SHELXTL (Sheldrick, 2008).

\section{(4-Bromophenyl)(morpholino)methanone (1b)}

Crystal data

Chemical formula $\quad \mathrm{C}_{11} \mathrm{H}_{12} \mathrm{BrNO}_{2}$

$M_{\mathrm{r}} \quad 270.13$

Crystal system, space Trigonal, $P 3_{1}$ group

Temperature $(\mathrm{K})$ 


$\begin{array}{ll}a, c(\AA) & 9.9577(2), 9.8213(2) \\ V\left(\AA^{3}\right) & 843.37(4) \\ Z & 3 \\ \text { Radiation type } & \text { Mo } K \alpha \\ \mu\left(\mathrm{mm}^{-1}\right) & 3.64 \\ \text { Crystal size }(\mathrm{mm}) & 0.5 \times 0.45 \times 0.4\end{array}$

Data collection

Diffractometer

Xcalibur

Absorption correction -

No. of measured,

$5805,2180,2129$

independent and

observed $[I>2 \sigma(I)]$

reflections

$\begin{array}{ll}R_{\text {int }} & 0.046 \\ (\sin \theta / \lambda)_{\max }\left(\AA^{-1}\right) & 0.617\end{array}$

Refinement

$R\left[F^{2}>2 \sigma\left(F^{2}\right)\right], w R\left(F^{2}\right), 0.023,0.057,1.05$

$S$

No. of reflections $\quad 2180$

No. of parameters $\quad 150$

No. of restraints 1

$\mathrm{H}$-atom treatment $\mathrm{H}$-atom parameters constrained

$\Delta \rho_{\max }, \Delta \rho_{\min }\left(\mathrm{e} \AA^{-3}\right) \quad 0.32,-0.37$

Absolute structure $\quad$ Flack $x$ determined using 1007 quotients $[(\mathrm{I}+)-(\mathrm{I}-)] /[(\mathrm{I}+)+(\mathrm{I}-)]$ (Parsons, Flack and Wagner, Acta Cryst. B69 (2013) 249-259).

Absolute structure $\quad-0.007$ (9)

parameter

Computer programs: CrysAlis CCD (Oxford Diffraction Ltd., 2008), SHELXS2014/7

(Sheldrick, 2014), SHELXL2014/7 (Sheldrick, 2014), SHELXTL (Sheldrick, 2008).

\section{(4-Chlorophenyl)(morpholino)methanone (1c)}

Crystal data

Chemical formula $\quad \mathrm{C}_{11} \mathrm{H}_{12} \mathrm{ClNO}_{2}$

$M_{\mathrm{r}} \quad 225.67$

Crystal system, space Trigonal, $P 3_{1}$

group

Temperature (K) $\quad 100$

$a, c(\AA)$

9.9808 (3), 9.5853 (3)

$V\left(\AA^{3}\right)$

$826.93(6)$

$Z$

3

Radiation type $\quad$ Mo $K \alpha$

$\mu\left(\mathrm{mm}^{-1}\right) \quad 0.33$ 
Crystal size $(\mathrm{mm}) \quad 0.5 \times 0.45 \times 0.4$

Data collection

Diffractometer

Xcalibur

Absorption correction -

No. of measured, $5673,1680,1590$

independent and

observed $[I>2 \sigma(I)]$

reflections

$\begin{array}{ll}R_{\text {int }} & 0.017 \\ (\sin \theta / \lambda)_{\max }\left(\AA^{-1}\right) & 0.616\end{array}$

Refinement

$R\left[F^{2}>2 \sigma\left(F^{2}\right)\right], w R\left(F^{2}\right), 0.028,0.068,1.09$

$S$

No. of reflections $\quad 1680$

No. of parameters $\quad 185$

No. of restraints 1

$\mathrm{H}$-atom treatment $\quad \mathrm{H}$-atom parameters constrained

$\Delta \rho_{\max }, \Delta \rho_{\min }\left(\mathrm{e} \AA^{-3}\right) \quad 0.27,-0.45$

Absolute structure $\quad$ Flack x determined using 505 quotients [(I+)-(I-)]/[(I+)+(I-)] (Parsons, Flack and Wagner, Acta Cryst. B69 (2013) 249-259).

Absolute structure $\quad 0.00(2)$

parameter

Computer programs: CrysAlis CCD (Oxford Diffraction Ltd., 2008), SHELXS2014/7

(Sheldrick, 2014), SHELXL2014/7 (Sheldrick, 2014), SHELXTL (Sheldrick, 2008).

\section{(4-Fluorophenyl)(morpholino)methanone (1d)}

Crystal data

Chemical formula

$M_{\mathrm{r}}$

$\mathrm{C}_{11} \mathrm{H}_{12} \mathrm{FNO}_{2}$

209.22

Crystal system, space group

Orthorhombic, $\mathrm{Pbca}$

Temperature $(\mathrm{K})$

100

$a, b, c(\AA)$

7.3584 (5), 7.2979 (5), 37.576 (2)

$V\left(\AA^{3}\right)$

2017.9 (2)

Z

8

Radiation type

Mo $K \alpha$

$\mu\left(\mathrm{mm}^{-1}\right)$

0.11

Crystal size (mm)

$0.2 \times 0.15 \times 0.1$

Data collection

Diffractometer

Xcalibur

Absorption correction 
No. of measured, independent 12441, 1977, 1193

and

observed $[I>2 \sigma(I)]$ reflections

$R_{\text {int }}$

$(\sin \theta / \lambda)_{\max }\left(\AA^{-1}\right)$

0.617

Refinement

$R\left[F^{2}>2 \sigma\left(F^{2}\right)\right], w R\left(F^{2}\right), S \quad 0.047,0.087,0.98$

No. of reflections 1977

No. of parameters

152

$\mathrm{H}$-atom treatment

$\mathrm{H}$ atoms treated by a mixture of independent and constrained refinement

$\Delta \rho_{\max }, \Delta \rho_{\min }\left(\mathrm{e} \AA^{-3}\right)$

$0.19,-0.23$

Computer programs: CrysAlis CCD (Oxford Diffraction Ltd., 2008), SHELXS2014/7 (Sheldrick, 2014), SHELXL2014/7 (Sheldrick, 2014), SHELXTL (Sheldrick, 2008).

Summary of selected geometric parameters $\left(\AA{ }^{\circ}\right)$ for $\mathbf{1 a - 1 d}$.

\begin{tabular}{|c|c|c|c|}
\hline \multicolumn{4}{|l|}{$1 \mathbf{a}$} \\
\hline $\mathrm{C} 1-\mathrm{C} 6$ & $1.384(3)$ & N9- C10 & $1.470(3)$ \\
\hline $\mathrm{C} 1-\mathrm{C} 2$ & $1.397(3)$ & $\mathrm{O} 8-\mathrm{C} 7$ & $1.234(2)$ \\
\hline $\mathrm{C} 1-\mathrm{H} 1$ & 0.9300 & $\mathrm{C} 10-\mathrm{C} 11$ & $1.515(3)$ \\
\hline $\mathrm{C} 2-\mathrm{C} 3$ & $1.394(3)$ & C10-H10A & 0.9700 \\
\hline $\mathrm{C} 2-\mathrm{C} 7$ & $1.496(3)$ & C10-H10B & 0.9700 \\
\hline $\mathrm{C} 3-\mathrm{C} 4$ & $1.386(3)$ & $\mathrm{C} 11-\mathrm{O} 12$ & $1.426(2)$ \\
\hline $\mathrm{C} 3-\mathrm{H} 3$ & 0.9300 & $\mathrm{C} 11-\mathrm{H} 11 \mathrm{~A}$ & 0.9700 \\
\hline $\mathrm{C} 4-\mathrm{C} 5$ & $1.390(3)$ & C11-H11B & 0.9700 \\
\hline $\mathrm{C} 4-\mathrm{H} 4$ & 0.9300 & $\mathrm{O} 12-\mathrm{C} 13$ & $1.430(2)$ \\
\hline $\mathrm{C} 5-\mathrm{C} 6$ & $1.391(3)$ & $\mathrm{C} 13-\mathrm{C} 14$ & $1.509(3)$ \\
\hline C5-I15 & $2.098(2)$ & $\mathrm{C} 13-\mathrm{H} 13 \mathrm{~A}$ & 0.9700 \\
\hline $\mathrm{C} 6-\mathrm{H} 6$ & 0.9300 & C13-H13B & 0.9700 \\
\hline N9-C7 & $1.357(2)$ & C14-H14A & 0.9700 \\
\hline N9-C14 & $1.469(2)$ & C14-H14B & 0.9700 \\
\hline $\mathrm{C} 6-\mathrm{C} 1-\mathrm{C} 2$ & $120.56(19)$ & $\mathrm{N} 9-\mathrm{C} 10-\mathrm{H} 10 \mathrm{~A}$ & |109.9 \\
\hline $\mathrm{C} 6-\mathrm{C} 1-\mathrm{H} 1$ & 119.7 & $\mathrm{C} 11-\mathrm{C} 10-\mathrm{H} 10 \mathrm{~A}$ & 109.9 \\
\hline $\mathrm{C} 2-\mathrm{C} 1-\mathrm{H} 1$ & 119.7 & N9- $\mathrm{C} 10-\mathrm{H} 10 \mathrm{~B}$ & 109.9 \\
\hline $\mathrm{C} 3-\mathrm{C} 2-\mathrm{C} 1$ & $119.1(2)$ & $\mathrm{C} 11-\mathrm{C} 10-\mathrm{H} 10 \mathrm{~B}$ & 109.9 \\
\hline $\mathrm{C} 3-\mathrm{C} 2-\mathrm{C} 7$ & $123.02(18)$ & $\mathrm{H} 10 \mathrm{~A}-\mathrm{C} 10-\mathrm{H} 10 \mathrm{~B}$ & 108.3 \\
\hline $\mathrm{C} 1-\mathrm{C} 2-\mathrm{C} 7$ & $117.72(18)$ & $\mathrm{O} 12-\mathrm{C} 11-\mathrm{C} 10$ & $111.76(17)$ \\
\hline
\end{tabular}




\begin{tabular}{|c|c|c|c|}
\hline $\mathrm{C} 4-\mathrm{C} 3-\mathrm{C} 2$ & $120.71(19)$ & $\mathrm{O} 12-\mathrm{C} 11-\mathrm{H} 11 \mathrm{~A}$ & 109.3 \\
\hline $\mathrm{C} 4-\mathrm{C} 3-\mathrm{H} 3$ & 119.6 & $\mathrm{C} 10-\mathrm{C} 11-\mathrm{H} 11 \mathrm{~A}$ & 109.3 \\
\hline $\mathrm{C} 2-\mathrm{C} 3-\mathrm{H} 3$ & 119.6 & $\mathrm{O} 12-\mathrm{C} 11-\mathrm{H} 11 \mathrm{~B}$ & 109.3 \\
\hline $\mathrm{C} 3-\mathrm{C} 4-\mathrm{C} 5$ & 119.52 (19) & $\mathrm{C} 10-\mathrm{C} 11-\mathrm{H} 11 \mathrm{~B}$ & 109.3 \\
\hline $\mathrm{C} 3-\mathrm{C} 4-\mathrm{H} 4$ & 120.2 & $\mathrm{H} 11 \mathrm{~A}-\mathrm{C} 11-\mathrm{H} 11 \mathrm{~B}$ & 107.9 \\
\hline $\mathrm{C} 5-\mathrm{C} 4-\mathrm{H} 4$ & 120.2 & $\mathrm{C} 11-\mathrm{O} 12-\mathrm{C} 13$ & $110.29(16)$ \\
\hline $\mathrm{C} 4-\mathrm{C} 5-\mathrm{C} 6$ & $120.44(19)$ & $\mathrm{O} 12-\mathrm{C} 13-\mathrm{C} 14$ & $111.01(17)$ \\
\hline C4-C5-I15 & $119.49(15)$ & $\mathrm{O} 12-\mathrm{C} 13-\mathrm{H} 13 \mathrm{~A}$ & 109.4 \\
\hline C6-C5-I15 & $120.07(16)$ & $\mathrm{C} 14-\mathrm{C} 13-\mathrm{H} 13 \mathrm{~A}$ & 109.4 \\
\hline $\mathrm{C} 1-\mathrm{C} 6-\mathrm{C} 5$ & $119.64(19)$ & $\mathrm{O} 12-\mathrm{C} 13-\mathrm{H} 13 \mathrm{~B}$ & 109.4 \\
\hline $\mathrm{C} 1-\mathrm{C} 6-\mathrm{H} 6$ & 120.2 & $\mathrm{C} 14-\mathrm{C} 13-\mathrm{H} 13 \mathrm{~B}$ & 109.4 \\
\hline $\mathrm{C} 5-\mathrm{C} 6-\mathrm{H} 6$ & 120.2 & $\mathrm{H} 13 \mathrm{~A}-\mathrm{C} 13-\mathrm{H} 13 \mathrm{~B}$ & 108.0 \\
\hline C7-N9-C14 & $126.04(17)$ & N9- $\mathrm{C} 14-\mathrm{C} 13$ & $109.70(17)$ \\
\hline C7-N9-C10 & $118.35(17)$ & N9- $\mathrm{C} 14-\mathrm{H} 14 \mathrm{~A}$ & 109.7 \\
\hline $\mathrm{C} 14-\mathrm{N} 9-\mathrm{C} 10$ & $112.91(16)$ & $\mathrm{C} 13-\mathrm{C} 14-\mathrm{H} 14 \mathrm{~A}$ & 109.7 \\
\hline $\mathrm{O} 8-\mathrm{C} 7-\mathrm{N} 9$ & $122.03(18)$ & N9- C14-H14B & 109.7 \\
\hline $\mathrm{O} 8-\mathrm{C} 7-\mathrm{C} 2$ & $119.18(18)$ & C13-C14-H14B & 109.7 \\
\hline $\mathrm{N} 9-\mathrm{C} 7-\mathrm{C} 2$ & $118.77(17)$ & $\mathrm{H} 14 \mathrm{~A}-\mathrm{C} 14-\mathrm{H} 14 \mathrm{~B}$ & 108.2 \\
\hline $\mathrm{N} 9-\mathrm{C} 10-\mathrm{C} 11$ & $109.13(16)$ & & \\
\hline $\mathrm{C} 6-\mathrm{C} 1-\mathrm{C} 2-\mathrm{C} 3$ & $-2.0(3)$ & $\mathrm{C} 10-\mathrm{N} 9-\mathrm{C} 7-\mathrm{C} 2$ & -176.19 (17) \\
\hline $\mathrm{C} 6-\mathrm{C} 1-\mathrm{C} 2-\mathrm{C} 7$ & $-177.07(18)$ & $\mathrm{C} 3-\mathrm{C} 2-\mathrm{C} 7-\mathrm{O} 8$ & $-132.8(2)$ \\
\hline $\mathrm{C} 1-\mathrm{C} 2-\mathrm{C} 3-\mathrm{C} 4$ & $1.1(3)$ & $\mathrm{C} 1-\mathrm{C} 2-\mathrm{C} 7-\mathrm{O} 8$ & $42.1(3)$ \\
\hline $\mathrm{C} 7-\mathrm{C} 2-\mathrm{C} 3-\mathrm{C} 4$ & $175.87(18)$ & $\mathrm{C} 3-\mathrm{C} 2-\mathrm{C} 7-\mathrm{N} 9$ & $45.5(3)$ \\
\hline $\mathrm{C} 2-\mathrm{C} 3-\mathrm{C} 4-\mathrm{C} 5$ & $1.2(3)$ & $\mathrm{C} 1-\mathrm{C} 2-\mathrm{C} 7-\mathrm{N} 9$ & $-139.69(19)$ \\
\hline $\mathrm{C} 3-\mathrm{C} 4-\mathrm{C} 5-\mathrm{C} 6$ & $-2.6(3)$ & $\mathrm{C} 7-\mathrm{N} 9-\mathrm{C} 10-\mathrm{C} 11$ & $144.61(18)$ \\
\hline $\mathrm{C} 3-\mathrm{C} 4-\mathrm{C} 5-\mathrm{I} 15$ & $177.17(14)$ & $\mathrm{C} 14-\mathrm{N} 9-\mathrm{C} 10-\mathrm{C} 11$ & $-52.9(2)$ \\
\hline $\mathrm{C} 2-\mathrm{C} 1-\mathrm{C} 6-\mathrm{C} 5$ & $0.7(3)$ & N9- $\mathrm{C} 10-\mathrm{C} 11-\mathrm{O} 12$ & $55.7(2)$ \\
\hline $\mathrm{C} 4-\mathrm{C} 5-\mathrm{C} 6-\mathrm{C} 1$ & $1.7(3)$ & $\mathrm{C} 10-\mathrm{C} 11-\mathrm{O} 12-\mathrm{C} 13$ & $-60.2(2)$ \\
\hline $\mathrm{I} 15-\mathrm{C} 5-\mathrm{C} 6-\mathrm{C} 1$ & $-178.08(15)$ & $\mathrm{C} 11-\mathrm{O} 12-\mathrm{C} 13-\mathrm{C} 14$ & $60.2(2)$ \\
\hline $\mathrm{C} 14-\mathrm{N} 9-\mathrm{C} 7-\mathrm{O} 8$ & $-158.01(19)$ & $\mathrm{C} 7-\mathrm{N} 9-\mathrm{C} 14-\mathrm{C} 13$ & $-145.45(19)$ \\
\hline $\mathrm{C} 10-\mathrm{N} 9-\mathrm{C} 7-\mathrm{O} 8$ & $2.0(3)$ & $\mathrm{C} 10-\mathrm{N} 9-\mathrm{C} 14-\mathrm{C} 13$ & $53.6(2)$ \\
\hline $\mathrm{C} 14-\mathrm{N} 9-\mathrm{C} 7-\mathrm{C} 2$ & $23.8(3)$ & $\mathrm{O} 12-\mathrm{C} 13-\mathrm{C} 14-\mathrm{N} 9$ & $-56.4(2)$ \\
\hline \multicolumn{4}{|l|}{$1 b$} \\
\hline $\mathrm{Br} 1-\mathrm{C} 4 \mathrm{P}$ & $1.902(4)$ & C5-H5A & 0.9700 \\
\hline $\mathrm{N} 1-\mathrm{C} 1$ & $1.345(5)$ & $\mathrm{C} 5-\mathrm{H} 5 \mathrm{~B}$ & 0.9700 \\
\hline
\end{tabular}




\begin{tabular}{|c|c|c|c|}
\hline $\mathrm{N} 1-\mathrm{C} 2$ & $1.467(5)$ & $\mathrm{C} 4-\mathrm{H} 4 \mathrm{~A}$ & 0.9700 \\
\hline $\mathrm{N} 1-\mathrm{C} 3$ & $1.468(5)$ & C4-H4B & 0.9700 \\
\hline $\mathrm{O} 1-\mathrm{C} 1$ & $1.231(4)$ & $\mathrm{C} 1 \mathrm{P}-\mathrm{C} 6 \mathrm{P}$ & $1.386(5)$ \\
\hline $\mathrm{C} 1-\mathrm{C} 1 \mathrm{P}$ & $1.506(5)$ & $\mathrm{C} 1 \mathrm{P}-\mathrm{C} 2 \mathrm{P}$ & $1.394(5)$ \\
\hline $\mathrm{C} 3-\mathrm{C} 5$ & $1.506(5)$ & $\mathrm{C} 2 \mathrm{P}-\mathrm{C} 3 \mathrm{P}$ & $1.383(5)$ \\
\hline $\mathrm{C} 3-\mathrm{H} 3 \mathrm{~A}$ & 0.9700 & $\mathrm{C} 2 \mathrm{P}-\mathrm{H} 2 \mathrm{P}$ & 0.9300 \\
\hline C3-H3B & 0.9700 & $\mathrm{C} 3 \mathrm{P}-\mathrm{C} 4 \mathrm{P}$ & $1.392(5)$ \\
\hline $\mathrm{C} 2-\mathrm{C} 4$ & $1.513(6)$ & $\mathrm{C} 3 \mathrm{P}-\mathrm{H} 3 \mathrm{P}$ & 0.9300 \\
\hline $\mathrm{C} 2-\mathrm{H} 2 \mathrm{~A}$ & 0.9700 & $\mathrm{C} 4 \mathrm{P}-\mathrm{C} 5 \mathrm{P}$ & $1.367(5)$ \\
\hline $\mathrm{C} 2-\mathrm{H} 2 \mathrm{~B}$ & 0.9700 & $\mathrm{C} 5 \mathrm{P}-\mathrm{C} 6 \mathrm{P}$ & $1.394(6)$ \\
\hline $\mathrm{O} 2-\mathrm{C} 5$ & $1.427(5)$ & $\mathrm{C} 5 \mathrm{P}-\mathrm{H} 5 \mathrm{P}$ & 0.9300 \\
\hline $\mathrm{O} 2-\mathrm{C} 4$ & $1.432(4)$ & C6P-H6P & 0.9300 \\
\hline $\mathrm{C} 1-\mathrm{N} 1-\mathrm{C} 2$ & 120.5 (3) & $\mathrm{O} 2-\mathrm{C} 4-\mathrm{C} 2$ & |111.9(3) \\
\hline $\mathrm{C} 1-\mathrm{N} 1-\mathrm{C} 3$ & $125.8(3)$ & $\mathrm{O} 2-\mathrm{C} 4-\mathrm{H} 4 \mathrm{~A}$ & 109.2 \\
\hline $\mathrm{C} 2-\mathrm{N} 1-\mathrm{C} 3$ & $113.2(3)$ & $\mathrm{C} 2-\mathrm{C} 4-\mathrm{H} 4 \mathrm{~A}$ & 109.2 \\
\hline $\mathrm{O} 1-\mathrm{C} 1-\mathrm{N} 1$ & $122.7(3)$ & $\mathrm{O} 2-\mathrm{C} 4-\mathrm{H} 4 \mathrm{~B}$ & 109.2 \\
\hline $\mathrm{O} 1-\mathrm{C} 1-\mathrm{C} 1 \mathrm{P}$ & $119.4(3)$ & $\mathrm{C} 2-\mathrm{C} 4-\mathrm{H} 4 \mathrm{~B}$ & 109.2 \\
\hline $\mathrm{N} 1-\mathrm{C} 1-\mathrm{C} 1 \mathrm{P}$ & $117.9(3)$ & $\mathrm{H} 4 \mathrm{~A}-\mathrm{C} 4-\mathrm{H} 4 \mathrm{~B}$ & 107.9 \\
\hline $\mathrm{N} 1-\mathrm{C} 3-\mathrm{C} 5$ & $108.9(3)$ & $\mathrm{C} 6 \mathrm{P}-\mathrm{C} 1 \mathrm{P}-\mathrm{C} 2 \mathrm{P}$ & $120.0(3)$ \\
\hline $\mathrm{N} 1-\mathrm{C} 3-\mathrm{H} 3 \mathrm{~A}$ & 109.9 & $\mathrm{C} 6 \mathrm{P}-\mathrm{C} 1 \mathrm{P}-\mathrm{C} 1$ & $122.2(3)$ \\
\hline $\mathrm{C} 5-\mathrm{C} 3-\mathrm{H} 3 \mathrm{~A}$ & 109.9 & $\mathrm{C} 2 \mathrm{P}-\mathrm{C} 1 \mathrm{P}-\mathrm{C} 1$ & $117.7(3)$ \\
\hline $\mathrm{N} 1-\mathrm{C} 3-\mathrm{H} 3 \mathrm{~B}$ & 109.9 & $\mathrm{C} 3 \mathrm{P}-\mathrm{C} 2 \mathrm{P}-\mathrm{C} 1 \mathrm{P}$ & $120.0(3)$ \\
\hline $\mathrm{C} 5-\mathrm{C} 3-\mathrm{H} 3 \mathrm{~B}$ & 109.9 & $\mathrm{C} 3 \mathrm{P}-\mathrm{C} 2 \mathrm{P}-\mathrm{H} 2 \mathrm{P}$ & 120.0 \\
\hline $\mathrm{H} 3 \mathrm{~A}-\mathrm{C} 3-\mathrm{H} 3 \mathrm{~B}$ & 108.3 & $\mathrm{C} 1 \mathrm{P}-\mathrm{C} 2 \mathrm{P}-\mathrm{H} 2 \mathrm{P}$ & 120.0 \\
\hline $\mathrm{N} 1-\mathrm{C} 2-\mathrm{C} 4$ & $110.1(3)$ & $\mathrm{C} 2 \mathrm{P}-\mathrm{C} 3 \mathrm{P}-\mathrm{C} 4 \mathrm{P}$ & $118.9(3)$ \\
\hline $\mathrm{N} 1-\mathrm{C} 2-\mathrm{H} 2 \mathrm{~A}$ & 109.6 & $\mathrm{C} 2 \mathrm{P}-\mathrm{C} 3 \mathrm{P}-\mathrm{H} 3 \mathrm{P}$ & 120.6 \\
\hline $\mathrm{C} 4-\mathrm{C} 2-\mathrm{H} 2 \mathrm{~A}$ & 109.6 & $\mathrm{C} 4 \mathrm{P}-\mathrm{C} 3 \mathrm{P}-\mathrm{H} 3 \mathrm{P}$ & 120.6 \\
\hline $\mathrm{N} 1-\mathrm{C} 2-\mathrm{H} 2 \mathrm{~B}$ & 109.6 & $\mathrm{C} 5 \mathrm{P}-\mathrm{C} 4 \mathrm{P}-\mathrm{C} 3 \mathrm{P}$ & $121.8(3)$ \\
\hline $\mathrm{C} 4-\mathrm{C} 2-\mathrm{H} 2 \mathrm{~B}$ & 109.6 & $\mathrm{C} 5 \mathrm{P}-\mathrm{C} 4 \mathrm{P}-\mathrm{Br} 1$ & $119.9(3)$ \\
\hline $\mathrm{H} 2 \mathrm{~A}-\mathrm{C} 2-\mathrm{H} 2 \mathrm{~B}$ & 108.2 & $\mathrm{C} 3 \mathrm{P}-\mathrm{C} 4 \mathrm{P}-\mathrm{Br} 1$ & $118.2(3)$ \\
\hline $\mathrm{C} 5-\mathrm{O} 2-\mathrm{C} 4$ & 109.8 (3) & $\mathrm{C} 4 \mathrm{P}-\mathrm{C} 5 \mathrm{P}-\mathrm{C} 6 \mathrm{P}$ & $119.1(3)$ \\
\hline $\mathrm{O} 2-\mathrm{C} 5-\mathrm{C} 3$ & $111.9(3)$ & $\mathrm{C} 4 \mathrm{P}-\mathrm{C} 5 \mathrm{P}-\mathrm{H} 5 \mathrm{P}$ & 120.4 \\
\hline $\mathrm{O} 2-\mathrm{C} 5-\mathrm{H} 5 \mathrm{~A}$ & 109.2 & $\mathrm{C} 6 \mathrm{P}-\mathrm{C} 5 \mathrm{P}-\mathrm{H} 5 \mathrm{P}$ & 120.4 \\
\hline $\mathrm{C} 3-\mathrm{C} 5-\mathrm{H} 5 \mathrm{~A}$ & 109.2 & $\mathrm{C} 1 \mathrm{P}-\mathrm{C} 6 \mathrm{P}-\mathrm{C} 5 \mathrm{P}$ & $120.0(3)$ \\
\hline $\mathrm{O} 2-\mathrm{C} 5-\mathrm{H} 5 \mathrm{~B}$ & 109.2 & $\mathrm{C} 1 \mathrm{P}-\mathrm{C} 6 \mathrm{P}-\mathrm{H} 6 \mathrm{P}$ & 120.0 \\
\hline C3- $55-\mathrm{H} 5 \mathrm{~B}$ & 109.2 & $\mathrm{C} 5 \mathrm{P}-\mathrm{C} 6 \mathrm{P}-\mathrm{H} 6 \mathrm{P}$ & 120.0 \\
\hline
\end{tabular}




\begin{tabular}{|c|c|c|c|}
\hline $\mathrm{H} 5 \mathrm{~A}-\mathrm{C} 5-\mathrm{H} 5 \mathrm{~B}$ & 107.9 & & \\
\hline $\mathrm{C} 2-\mathrm{N} 1-\mathrm{C} 1-\mathrm{O} 1$ & $3.2(5)$ & $\mathrm{N} 1-\mathrm{C} 1-\mathrm{C} 1 \mathrm{P}-\mathrm{C} 6 \mathrm{P}$ & $-76.7(4)$ \\
\hline $\mathrm{C} 3-\mathrm{N} 1-\mathrm{C} 1-\mathrm{O} 1$ & $175.0(3)$ & $\mathrm{O} 1-\mathrm{C} 1-\mathrm{C} 1 \mathrm{P}-\mathrm{C} 2 \mathrm{P}$ & $-72.0(4)$ \\
\hline $\mathrm{C} 2-\mathrm{N} 1-\mathrm{C} 1-\mathrm{C} 1 \mathrm{P}$ & $-175.4(3)$ & $\mathrm{N} 1-\mathrm{C} 1-\mathrm{C} 1 \mathrm{P}-\mathrm{C} 2 \mathrm{P}$ & 106.7 (4) \\
\hline $\mathrm{C} 3-\mathrm{N} 1-\mathrm{C} 1-\mathrm{C} 1 \mathrm{P}$ & $-3.5(5)$ & $\mathrm{C} 6 \mathrm{P}-\mathrm{C} 1 \mathrm{P}-\mathrm{C} 2 \mathrm{P}-\mathrm{C} 3 \mathrm{P}$ & $2.7(5)$ \\
\hline $\mathrm{C} 1-\mathrm{N} 1-\mathrm{C} 3-\mathrm{C} 5$ & $134.5(4)$ & $\mathrm{C} 1-\mathrm{C} 1 \mathrm{P}-\mathrm{C} 2 \mathrm{P}-\mathrm{C} 3 \mathrm{P}$ & $179.5(3)$ \\
\hline $\mathrm{C} 2-\mathrm{N} 1-\mathrm{C} 3-\mathrm{C} 5$ & $-53.1(4)$ & $\mathrm{C} 1 \mathrm{P}-\mathrm{C} 2 \mathrm{P}-\mathrm{C} 3 \mathrm{P}-\mathrm{C} 4 \mathrm{P}$ & $-1.8(5)$ \\
\hline $\mathrm{C} 1-\mathrm{N} 1-\mathrm{C} 2-\mathrm{C} 4$ & $-135.4(3)$ & $\mathrm{C} 2 \mathrm{P}-\mathrm{C} 3 \mathrm{P}-\mathrm{C} 4 \mathrm{P}-\mathrm{C} 5 \mathrm{P}$ & $-0.7(5)$ \\
\hline $\mathrm{C} 3-\mathrm{N} 1-\mathrm{C} 2-\mathrm{C} 4$ & $51.8(4)$ & $\mathrm{C} 2 \mathrm{P}-\mathrm{C} 3 \mathrm{P}-\mathrm{C} 4 \mathrm{P}-\mathrm{Br} 1$ & $176.4(3)$ \\
\hline $\mathrm{C} 4-\mathrm{O} 2-\mathrm{C} 5-\mathrm{C} 3$ & $-60.9(4)$ & $\mathrm{C} 3 \mathrm{P}-\mathrm{C} 4 \mathrm{P}-\mathrm{C} 5 \mathrm{P}-\mathrm{C} 6 \mathrm{P}$ & $2.1(6)$ \\
\hline $\mathrm{N} 1-\mathrm{C} 3-\mathrm{C} 5-\mathrm{O} 2$ & $57.4(4)$ & $\mathrm{Br} 1-\mathrm{C} 4 \mathrm{P}-\mathrm{C} 5 \mathrm{P}-\mathrm{C} 6 \mathrm{P}$ & $-174.9(3)$ \\
\hline $\mathrm{C} 5-\mathrm{O} 2-\mathrm{C} 4-\mathrm{C} 2$ & $58.8(4)$ & $\mathrm{C} 2 \mathrm{P}-\mathrm{C} 1 \mathrm{P}-\mathrm{C} 6 \mathrm{P}-\mathrm{C} 5 \mathrm{P}$ & $-1.3(6)$ \\
\hline $\mathrm{N} 1-\mathrm{C} 2-\mathrm{C} 4-\mathrm{O} 2$ & $-54.0(4)$ & $\mathrm{C} 1-\mathrm{C} 1 \mathrm{P}-\mathrm{C} 6 \mathrm{P}-\mathrm{C} 5 \mathrm{P}$ & $-177.9(3)$ \\
\hline $\mathrm{O} 1-\mathrm{C} 1-\mathrm{C} 1 \mathrm{P}-\mathrm{C} 6 \mathrm{P}$ & 104.7 (4) & $\mathrm{C} 4 \mathrm{P}-\mathrm{C} 5 \mathrm{P}-\mathrm{C} 6 \mathrm{P}-\mathrm{C} 1 \mathrm{P}$ & $-1.1(6)$ \\
\hline \multicolumn{4}{|l|}{$1 \mathrm{c}$} \\
\hline $\mathrm{C} 1-\mathrm{C} 6$ & $1.388(4)$ & C10A-H10A & 0.9700 \\
\hline $\mathrm{C} 1-\mathrm{C} 2$ & $1.391(3)$ & $\mathrm{C} 10 \mathrm{~A}-\mathrm{H} 10 \mathrm{~B}$ & 0.9700 \\
\hline $\mathrm{C} 1-\mathrm{C} 7$ & $1.503(3)$ & $\mathrm{C} 11 \mathrm{~A}-\mathrm{H} 11 \mathrm{~A}$ & 0.9700 \\
\hline $\mathrm{C} 2-\mathrm{C} 3$ & 1.387 (4) & $\mathrm{C} 11 \mathrm{~A}-\mathrm{H} 11 \mathrm{~B}$ & 0.9700 \\
\hline $\mathrm{C} 2-\mathrm{H} 2$ & 0.9300 & $\mathrm{C} 13 \mathrm{~A}-\mathrm{C} 14 \mathrm{~A}$ & $1.518(6)$ \\
\hline $\mathrm{C} 3-\mathrm{C} 4$ & $1.375(4)$ & $\mathrm{C} 13 \mathrm{~A}-\mathrm{H} 13 \mathrm{~A}$ & 0.9700 \\
\hline $\mathrm{C} 3-\mathrm{H} 3$ & 0.9300 & $\mathrm{C} 13 \mathrm{~A}-\mathrm{H} 13 \mathrm{~B}$ & 0.9700 \\
\hline $\mathrm{C} 4-\mathrm{C} 5$ & $1.388(4)$ & $\mathrm{C} 14 \mathrm{~A}-\mathrm{H} 14 \mathrm{~A}$ & 0.9700 \\
\hline $\mathrm{C} 4-\mathrm{Cl15}$ & $1.741(3)$ & $\mathrm{C} 14 \mathrm{~A}-\mathrm{H} 14 \mathrm{~B}$ & 0.9700 \\
\hline $\mathrm{C} 5-\mathrm{C} 6$ & $1.381(4)$ & $\mathrm{N} 9 \mathrm{~A}-\mathrm{C} 14 \mathrm{~B}$ & $1.466(10)$ \\
\hline C5-H5 & 0.9300 & $\mathrm{~N} 9 \mathrm{~A}-\mathrm{C} 10 \mathrm{~B}$ & $1.482(10)$ \\
\hline $\mathrm{C} 6-\mathrm{H} 6$ & 0.9300 & $\mathrm{C} 10 \mathrm{~B}-\mathrm{C} 11 \mathrm{~B}$ & $1.489(12)$ \\
\hline $\mathrm{C} 7-\mathrm{O} 8$ & $1.224(3)$ & $\mathrm{C} 10 \mathrm{~B}-\mathrm{H} 10 \mathrm{C}$ & 0.9700 \\
\hline C7-N9A & $1.334(8)$ & $\mathrm{C} 10 \mathrm{~B}-\mathrm{H} 10 \mathrm{D}$ & 0.9700 \\
\hline C7-N9B & $1.389(5)$ & $\mathrm{C} 11 \mathrm{~B}-\mathrm{H} 11 \mathrm{C}$ & 0.9700 \\
\hline $\mathrm{O} 12-\mathrm{C} 13 \mathrm{~A}$ & $1.418(6)$ & C11B-H11D & 0.9700 \\
\hline $\mathrm{O} 12-\mathrm{C} 11 \mathrm{~A}$ & $1.444(5)$ & $\mathrm{C} 13 \mathrm{~B}-\mathrm{C} 14 \mathrm{~B}$ & $1.474(14)$ \\
\hline $\mathrm{O} 12-\mathrm{C} 11 \mathrm{~B}$ & $1.490(8)$ & $\mathrm{C} 13 \mathrm{~B}-\mathrm{H} 13 \mathrm{C}$ & 0.9700 \\
\hline $\mathrm{O} 12-\mathrm{C} 13 \mathrm{~B}$ & $1.516(10)$ & $\mathrm{C} 13 \mathrm{~B}-\mathrm{H} 13 \mathrm{D}$ & 0.9700 \\
\hline $\mathrm{N} 9 \mathrm{~B}-\mathrm{C} 10 \mathrm{~A}$ & $1.463(5)$ & $\mathrm{C} 14 \mathrm{~B}-\mathrm{H} 14 \mathrm{C}$ & 0.9700 \\
\hline
\end{tabular}




\begin{tabular}{|c|c|c|c|}
\hline N9B-C14A & $1.465(6)$ & C14B-H14D & 0.9700 \\
\hline $\mathrm{C} 10 \mathrm{~A}-\mathrm{C} 11 \mathrm{~A}$ & $1.501(6)$ & & \\
\hline $\mathrm{C} 6-\mathrm{C} 1-\mathrm{C} 2$ & |119.7 (2) & $\mathrm{O} 12-\mathrm{C} 13 \mathrm{~A}-\mathrm{C} 14 \mathrm{~A}$ & |108.8 (4) \\
\hline $\mathrm{C} 6-\mathrm{C} 1-\mathrm{C} 7$ & $117.9(2)$ & $\mathrm{O} 12-\mathrm{C} 13 \mathrm{~A}-\mathrm{H} 13 \mathrm{~A}$ & 109.9 \\
\hline $\mathrm{C} 2-\mathrm{C} 1-\mathrm{C} 7$ & $122.2(2)$ & $\mathrm{C} 14 \mathrm{~A}-\mathrm{C} 13 \mathrm{~A}-\mathrm{H} 13 \mathrm{~A}$ & 109.9 \\
\hline $\mathrm{C} 3-\mathrm{C} 2-\mathrm{C} 1$ & $120.1(3)$ & $\mathrm{O} 12-\mathrm{C} 13 \mathrm{~A}-\mathrm{H} 13 \mathrm{~B}$ & 109.9 \\
\hline $\mathrm{C} 3-\mathrm{C} 2-\mathrm{H} 2$ & 119.9 & $\mathrm{C} 14 \mathrm{~A}-\mathrm{C} 13 \mathrm{~A}-\mathrm{H} 13 \mathrm{~B}$ & 109.9 \\
\hline $\mathrm{C} 1-\mathrm{C} 2-\mathrm{H} 2$ & 119.9 & $\mathrm{H} 13 \mathrm{~A}-\mathrm{C} 13 \mathrm{~A}-\mathrm{H} 13 \mathrm{~B}$ & 108.3 \\
\hline $\mathrm{C} 4-\mathrm{C} 3-\mathrm{C} 2$ & $119.0(2)$ & $\mathrm{N} 9 \mathrm{~B}-\mathrm{C} 14 \mathrm{~A}-\mathrm{C} 13 \mathrm{~A}$ & $109.7(4)$ \\
\hline $\mathrm{C} 4-\mathrm{C} 3-\mathrm{H} 3$ & 120.5 & $\mathrm{~N} 9 \mathrm{~B}-\mathrm{C} 14 \mathrm{~A}-\mathrm{H} 14 \mathrm{~A}$ & 109.7 \\
\hline $\mathrm{C} 2-\mathrm{C} 3-\mathrm{H} 3$ & 120.5 & $\mathrm{C} 13 \mathrm{~A}-\mathrm{C} 14 \mathrm{~A}-\mathrm{H} 14 \mathrm{~A}$ & 109.7 \\
\hline $\mathrm{C} 3-\mathrm{C} 4-\mathrm{C} 5$ & $121.8(3)$ & $\mathrm{N} 9 \mathrm{~B}-\mathrm{C} 14 \mathrm{~A}-\mathrm{H} 14 \mathrm{~B}$ & 109.7 \\
\hline $\mathrm{C} 3-\mathrm{C} 4-\mathrm{Cl} 15$ & $120.0(2)$ & $\mathrm{C} 13 \mathrm{~A}-\mathrm{C} 14 \mathrm{~A}-\mathrm{H} 14 \mathrm{~B}$ & 109.7 \\
\hline $\mathrm{C} 5-\mathrm{C} 4-\mathrm{Cl} 15$ & $118.2(2)$ & $\mathrm{H} 14 \mathrm{~A}-\mathrm{C} 14 \mathrm{~A}-\mathrm{H} 14 \mathrm{~B}$ & 108.2 \\
\hline $\mathrm{C} 6-\mathrm{C} 5-\mathrm{C} 4$ & $118.6(3)$ & C7-N9A-C14B & $126.4(6)$ \\
\hline $\mathrm{C} 6-\mathrm{C} 5-\mathrm{H} 5$ & 120.7 & C7-N9A-C10B & $120.2(6)$ \\
\hline $\mathrm{C} 4-\mathrm{C} 5-\mathrm{H} 5$ & 120.7 & $\mathrm{C} 14 \mathrm{~B}-\mathrm{N} 9 \mathrm{~A}-\mathrm{C} 10 \mathrm{~B}$ & $113.2(6)$ \\
\hline $\mathrm{C} 5-\mathrm{C} 6-\mathrm{C} 1$ & $120.6(2)$ & $\mathrm{N} 9 \mathrm{~A}-\mathrm{C} 10 \mathrm{~B}-\mathrm{C} 11 \mathrm{~B}$ & $109.8(6)$ \\
\hline $\mathrm{C} 5-\mathrm{C} 6-\mathrm{H} 6$ & 119.7 & $\mathrm{~N} 9 \mathrm{~A}-\mathrm{C} 10 \mathrm{~B}-\mathrm{H} 10 \mathrm{C}$ & 109.7 \\
\hline $\mathrm{C} 1-\mathrm{C} 6-\mathrm{H} 6$ & 119.7 & $\mathrm{C} 11 \mathrm{~B}-\mathrm{C} 10 \mathrm{~B}-\mathrm{H} 10 \mathrm{C}$ & 109.7 \\
\hline $\mathrm{O} 8-\mathrm{C} 7-\mathrm{N} 9 \mathrm{~A}$ & $120.0(4)$ & $\mathrm{N} 9 \mathrm{~A}-\mathrm{C} 10 \mathrm{~B}-\mathrm{H} 10 \mathrm{D}$ & 109.7 \\
\hline $\mathrm{O} 8-\mathrm{C} 7-\mathrm{N} 9 \mathrm{~B}$ & $121.6(3)$ & $\mathrm{C} 11 \mathrm{~B}-\mathrm{C} 10 \mathrm{~B}-\mathrm{H} 10 \mathrm{D}$ & 109.7 \\
\hline $\mathrm{O} 8-\mathrm{C} 7-\mathrm{C} 1$ & $119.5(2)$ & $\mathrm{H} 10 \mathrm{C}-\mathrm{C} 10 \mathrm{~B}-\mathrm{H} 10 \mathrm{D}$ & 108.2 \\
\hline $\mathrm{N} 9 \mathrm{~A}-\mathrm{C} 7-\mathrm{C} 1$ & $116.1(4)$ & $\mathrm{C} 10 \mathrm{~B}-\mathrm{C} 11 \mathrm{~B}-\mathrm{O} 12$ & $109.4(6)$ \\
\hline $\mathrm{N} 9 \mathrm{~B}-\mathrm{C} 7-\mathrm{C} 1$ & $118.1(2)$ & $\mathrm{C} 10 \mathrm{~B}-\mathrm{C} 11 \mathrm{~B}-\mathrm{H} 11 \mathrm{C}$ & 109.8 \\
\hline $\mathrm{C} 13 \mathrm{~A}-\mathrm{O} 12-\mathrm{C} 11 \mathrm{~A}$ & $110.6(3)$ & $\mathrm{O} 12-\mathrm{C} 11 \mathrm{~B}-\mathrm{H} 11 \mathrm{C}$ & 109.8 \\
\hline $\mathrm{C} 11 \mathrm{~B}-\mathrm{O} 12-\mathrm{C} 13 \mathrm{~B}$ & $102.8(5)$ & $\mathrm{C} 10 \mathrm{~B}-\mathrm{C} 11 \mathrm{~B}-\mathrm{H} 11 \mathrm{D}$ & 109.8 \\
\hline $\mathrm{C} 7-\mathrm{N} 9 \mathrm{~B}-\mathrm{C} 10 \mathrm{~A}$ & $121.0(3)$ & $\mathrm{O} 12-\mathrm{C} 11 \mathrm{~B}-\mathrm{H} 11 \mathrm{D}$ & 109.8 \\
\hline $\mathrm{C} 7-\mathrm{N} 9 \mathrm{~B}-\mathrm{C} 14 \mathrm{~A}$ & $126.0(3)$ & $\mathrm{H} 11 \mathrm{C}-\mathrm{C} 11 \mathrm{~B}-\mathrm{H} 11 \mathrm{D}$ & 108.2 \\
\hline $\mathrm{C} 10 \mathrm{~A}-\mathrm{N} 9 \mathrm{~B}-\mathrm{C} 14 \mathrm{~A}$ & $113.0(4)$ & $\mathrm{C} 14 \mathrm{~B}-\mathrm{C} 13 \mathrm{~B}-\mathrm{O} 12$ & $108.7(7)$ \\
\hline $\mathrm{N} 9 \mathrm{~B}-\mathrm{C} 10 \mathrm{~A}-\mathrm{C} 11 \mathrm{~A}$ & $110.7(3)$ & $\mathrm{C} 14 \mathrm{~B}-\mathrm{C} 13 \mathrm{~B}-\mathrm{H} 13 \mathrm{C}$ & 110.0 \\
\hline $\mathrm{N} 9 \mathrm{~B}-\mathrm{C} 10 \mathrm{~A}-\mathrm{H} 10 \mathrm{~A}$ & 109.5 & $\mathrm{O} 12-\mathrm{C} 13 \mathrm{~B}-\mathrm{H} 13 \mathrm{C}$ & 110.0 \\
\hline $\mathrm{C} 11 \mathrm{~A}-\mathrm{C} 10 \mathrm{~A}-\mathrm{H} 10 \mathrm{~A}$ & 109.5 & $\mathrm{C} 14 \mathrm{~B}-\mathrm{C} 13 \mathrm{~B}-\mathrm{H} 13 \mathrm{D}$ & 110.0 \\
\hline $\mathrm{N} 9 \mathrm{~B}-\mathrm{C} 10 \mathrm{~A}-\mathrm{H} 10 \mathrm{~B}$ & 109.5 & $\mathrm{O} 12-\mathrm{C} 13 \mathrm{~B}-\mathrm{H} 13 \mathrm{D}$ & 110.0 \\
\hline $\mathrm{C} 11 \mathrm{~A}-\mathrm{C} 10 \mathrm{~A}-\mathrm{H} 10 \mathrm{~B}$ & 109.5 & $\mathrm{H} 13 \mathrm{C}-\mathrm{C} 13 \mathrm{~B}-\mathrm{H} 13 \mathrm{D}$ & 108.3 \\
\hline $\mathrm{H} 10 \mathrm{~A}-\mathrm{C} 10 \mathrm{~A}-\mathrm{H} 10 \mathrm{~B}$ & 108.1 & N9A $-\mathrm{C} 14 \mathrm{~B}-\mathrm{C} 13 \mathrm{~B}$ & $109.8(7)$ \\
\hline
\end{tabular}




\begin{tabular}{|c|c|c|c|}
\hline $\mathrm{O} 12-\mathrm{C} 11 \mathrm{~A}-\mathrm{C} 10 \mathrm{~A}$ & $108.3(3)$ & N9A-C14B-H14C & 109.7 \\
\hline $\mathrm{O} 12-\mathrm{C} 11 \mathrm{~A}-\mathrm{H} 11 \mathrm{~A}$ & 110.0 & $\mathrm{C} 13 \mathrm{~B}-\mathrm{C} 14 \mathrm{~B}-\mathrm{H} 14 \mathrm{C}$ & 109.7 \\
\hline $\mathrm{C} 10 \mathrm{~A}-\mathrm{C} 11 \mathrm{~A}-\mathrm{H} 11 \mathrm{~A}$ & 110.0 & N9A-C14B-H14D & 109.7 \\
\hline $\mathrm{O} 12-\mathrm{C} 11 \mathrm{~A}-\mathrm{H} 11 \mathrm{~B}$ & 110.0 & $\mathrm{C} 13 \mathrm{~B}-\mathrm{C} 14 \mathrm{~B}-\mathrm{H} 14 \mathrm{D}$ & 109.7 \\
\hline $\mathrm{C} 10 \mathrm{~A}-\mathrm{C} 11 \mathrm{~A}-\mathrm{H} 11 \mathrm{~B}$ & 110.0 & $\mathrm{H} 14 \mathrm{C}-\mathrm{C} 14 \mathrm{~B}-\mathrm{H} 14 \mathrm{D}$ & 108.2 \\
\hline $\mathrm{H} 11 \mathrm{~A}-\mathrm{C} 11 \mathrm{~A}-\mathrm{H} 11 \mathrm{~B}$ & 108.4 & & \\
\hline $\mathrm{C} 6-\mathrm{C} 1-\mathrm{C} 2-\mathrm{C} 3$ & $\mid-0.9(4)$ & $\mathrm{C} 7-\mathrm{N} 9 \mathrm{~B}-\mathrm{C} 10 \mathrm{~A}-\mathrm{C} 11 \mathrm{~A}$ & $126.8(4)$ \\
\hline $\mathrm{C} 7-\mathrm{C} 1-\mathrm{C} 2-\mathrm{C} 3$ & $-175.8(2)$ & $\mathrm{C} 14 \mathrm{~A}-\mathrm{N} 9 \mathrm{~B}-\mathrm{C} 10 \mathrm{~A}-\mathrm{C} 11 \mathrm{~A}$ & $-52.4(5)$ \\
\hline $\mathrm{C} 1-\mathrm{C} 2-\mathrm{C} 3-\mathrm{C} 4$ & $-1.2(4)$ & $\mathrm{C} 13 \mathrm{~A}-\mathrm{O} 12-\mathrm{C} 11 \mathrm{~A}-\mathrm{C} 10 \mathrm{~A}$ & $-64.4(5)$ \\
\hline $\mathrm{C} 2-\mathrm{C} 3-\mathrm{C} 4-\mathrm{C} 5$ & $1.8(4)$ & $\mathrm{N} 9 \mathrm{~B}-\mathrm{C} 10 \mathrm{~A}-\mathrm{C} 11 \mathrm{~A}-\mathrm{O} 12$ & $56.5(5)$ \\
\hline $\mathrm{C} 2-\mathrm{C} 3-\mathrm{C} 4-\mathrm{Cl} 15$ & $-177.10(19)$ & $\mathrm{C} 11 \mathrm{~A}-\mathrm{O} 12-\mathrm{C} 13 \mathrm{~A}-\mathrm{C} 14 \mathrm{~A}$ & $64.7(5)$ \\
\hline $\mathrm{C} 3-\mathrm{C} 4-\mathrm{C} 5-\mathrm{C} 6$ & $-0.3(4)$ & $\mathrm{C} 7-\mathrm{N} 9 \mathrm{~B}-\mathrm{C} 14 \mathrm{~A}-\mathrm{C} 13 \mathrm{~A}$ & $-127.2(4)$ \\
\hline $\mathrm{Cl} 15-\mathrm{C} 4-\mathrm{C} 5-\mathrm{C} 6$ & $178.56(19)$ & $\mathrm{C} 10 \mathrm{~A}-\mathrm{N} 9 \mathrm{~B}-\mathrm{C} 14 \mathrm{~A}-\mathrm{C} 13 \mathrm{~A}$ & $52.0(5)$ \\
\hline $\mathrm{C} 4-\mathrm{C} 5-\mathrm{C} 6-\mathrm{C} 1$ & $-1.7(3)$ & $\mathrm{O} 12-\mathrm{C} 13 \mathrm{~A}-\mathrm{C} 14 \mathrm{~A}-\mathrm{N} 9 \mathrm{~B}$ & $-57.1(5)$ \\
\hline $\mathrm{C} 2-\mathrm{C} 1-\mathrm{C} 6-\mathrm{C} 5$ & $2.3(4)$ & $\mathrm{O} 8-\mathrm{C} 7-\mathrm{N} 9 \mathrm{~A}-\mathrm{C} 14 \mathrm{~B}$ & $169.4(6)$ \\
\hline $\mathrm{C} 7-\mathrm{C} 1-\mathrm{C} 6-\mathrm{C} 5$ & $177.5(2)$ & $\mathrm{C} 1-\mathrm{C} 7-\mathrm{N} 9 \mathrm{~A}-\mathrm{C} 14 \mathrm{~B}$ & $13.0(10)$ \\
\hline $\mathrm{C} 6-\mathrm{C} 1-\mathrm{C} 7-\mathrm{O} 8$ & $-64.8(3)$ & $\mathrm{O} 8-\mathrm{C} 7-\mathrm{N} 9 \mathrm{~A}-\mathrm{C} 10 \mathrm{~B}$ & $-16.7(9)$ \\
\hline $\mathrm{C} 2-\mathrm{C} 1-\mathrm{C} 7-\mathrm{O} 8$ & $110.2(3)$ & $\mathrm{C} 1-\mathrm{C} 7-\mathrm{N} 9 \mathrm{~A}-\mathrm{C} 10 \mathrm{~B}$ & $-173.1(6)$ \\
\hline $\mathrm{C} 6-\mathrm{C} 1-\mathrm{C} 7-\mathrm{N} 9 \mathrm{~A}$ & $91.7(5)$ & $\mathrm{C} 7-\mathrm{N} 9 \mathrm{~A}-\mathrm{C} 10 \mathrm{~B}-\mathrm{C} 11 \mathrm{~B}$ & $-123.5(8)$ \\
\hline $\mathrm{C} 2-\mathrm{C} 1-\mathrm{C} 7-\mathrm{N} 9 \mathrm{~A}$ & $-93.3(5)$ & $\mathrm{C} 14 \mathrm{~B}-\mathrm{N} 9 \mathrm{~A}-\mathrm{C} 10 \mathrm{~B}-\mathrm{C} 11 \mathrm{~B}$ & $51.1(9)$ \\
\hline $\mathrm{C} 6-\mathrm{C} 1-\mathrm{C} 7-\mathrm{N} 9 \mathrm{~B}$ & $124.7(3)$ & $\mathrm{N} 9 \mathrm{~A}-\mathrm{C} 10 \mathrm{~B}-\mathrm{C} 11 \mathrm{~B}-\mathrm{O} 12$ & $-60.1(8)$ \\
\hline $\mathrm{C} 2-\mathrm{C} 1-\mathrm{C} 7-\mathrm{N} 9 \mathrm{~B}$ & $-60.2(3)$ & $\mathrm{C} 13 \mathrm{~B}-\mathrm{O} 12-\mathrm{C} 11 \mathrm{~B}-\mathrm{C} 10 \mathrm{~B}$ & $67.7(8)$ \\
\hline $\mathrm{O} 8-\mathrm{C} 7-\mathrm{N} 9 \mathrm{~B}-\mathrm{C} 10 \mathrm{~A}$ & $6.7(5)$ & $\mathrm{C} 11 \mathrm{~B}-\mathrm{O} 12-\mathrm{C} 13 \mathrm{~B}-\mathrm{C} 14 \mathrm{~B}$ & $-68.8(8)$ \\
\hline $\mathrm{C} 1-\mathrm{C} 7-\mathrm{N} 9 \mathrm{~B}-\mathrm{C} 10 \mathrm{~A}$ & $176.9(3)$ & $\mathrm{C} 7-\mathrm{N} 9 \mathrm{~A}-\mathrm{C} 14 \mathrm{~B}-\mathrm{C} 13 \mathrm{~B}$ & $121.7(9)$ \\
\hline $\mathrm{O} 8-\mathrm{C} 7-\mathrm{N} 9 \mathrm{~B}-\mathrm{C} 14 \mathrm{~A}$ & $-174.2(3)$ & $\mathrm{C} 10 \mathrm{~B}-\mathrm{N} 9 \mathrm{~A}-\mathrm{C} 14 \mathrm{~B}-\mathrm{C} 13 \mathrm{~B}$ & $-52.6(9)$ \\
\hline $\mathrm{C} 1-\mathrm{C} 7-\mathrm{N} 9 \mathrm{~B}-\mathrm{C} 14 \mathrm{~A}$ & $-3.9(5)$ & $\mathrm{O} 12-\mathrm{C} 13 \mathrm{~B}-\mathrm{C} 14 \mathrm{~B}-\mathrm{N} 9 \mathrm{~A}$ & $62.1(9)$ \\
\hline \multicolumn{4}{|l|}{ 1d } \\
\hline $\mathrm{C} 1-\mathrm{C} 6$ & $1.385(3)$ & $\mathrm{N} 9-\mathrm{C} 14$ & $1.463(2)$ \\
\hline $\mathrm{C} 1-\mathrm{C} 2$ & $1.391(3)$ & N9- $\mathrm{C} 10$ & $1.470(2)$ \\
\hline $\mathrm{C} 1-\mathrm{C} 7$ & $1.501(3)$ & $\mathrm{C} 10-\mathrm{C} 11$ & $1.513(3)$ \\
\hline $\mathrm{C} 2-\mathrm{C} 3$ & $1.381(3)$ & $\mathrm{C} 10-\mathrm{H} 10 \mathrm{~A}$ & 0.9700 \\
\hline $\mathrm{C} 2-\mathrm{H} 2$ & 0.9300 & C10-H10B & 0.9700 \\
\hline $\mathrm{C} 3-\mathrm{C} 4$ & $1.370(3)$ & $\mathrm{C} 11-\mathrm{O} 12$ & $1.430(2)$ \\
\hline $\mathrm{C} 3-\mathrm{H} 3$ & 0.9300 & C11-H11A & 0.9700 \\
\hline $\mathrm{C} 4-\mathrm{C} 5$ & $1.366(3)$ & C11-H11B & 0.9700 \\
\hline
\end{tabular}




\begin{tabular}{|c|c|c|c|}
\hline $\mathrm{C} 4-\mathrm{F} 15$ & $1.366(2)$ & $\mathrm{O} 12-\mathrm{C} 13$ & $1.429(2)$ \\
\hline $\mathrm{C} 5-\mathrm{C} 6$ & $1.386(3)$ & $\mathrm{C} 13-\mathrm{C} 14$ & $1.508(3)$ \\
\hline $\mathrm{C} 5-\mathrm{H} 5$ & 0.9300 & C13-H13A & 0.9700 \\
\hline $\mathrm{C} 6-\mathrm{H} 6$ & 0.9300 & C13-H13B & 0.9700 \\
\hline $\mathrm{O} 8-\mathrm{C} 7$ & $1.236(2)$ & C14-H14A & 0.9700 \\
\hline C7-N9 & 1.354 (2) & C14-H14B & 0.9700 \\
\hline $\mathrm{C} 6-\mathrm{C} 1-\mathrm{C} 2$ & |119.18 (18) & $\mathrm{N} 9-\mathrm{C} 10-\mathrm{H} 10 \mathrm{~A}$ & 109.8 \\
\hline $\mathrm{C} 6-\mathrm{C} 1-\mathrm{C} 7$ & $118.88(18)$ & $\mathrm{C} 11-\mathrm{C} 10-\mathrm{H} 10 \mathrm{~A}$ & 109.8 \\
\hline $\mathrm{C} 2-\mathrm{C} 1-\mathrm{C} 7$ & $121.81(18)$ & N9- $\mathrm{C} 10-\mathrm{H} 10 \mathrm{~B}$ & 109.8 \\
\hline $\mathrm{C} 3-\mathrm{C} 2-\mathrm{C} 1$ & $120.6(2)$ & $\mathrm{C} 11-\mathrm{C} 10-\mathrm{H} 10 \mathrm{~B}$ & 109.8 \\
\hline $\mathrm{C} 3-\mathrm{C} 2-\mathrm{H} 2$ & 119.7 & $\mathrm{H} 10 \mathrm{~A}-\mathrm{C} 10-\mathrm{H} 10 \mathrm{~B}$ & 108.2 \\
\hline $\mathrm{C} 1-\mathrm{C} 2-\mathrm{H} 2$ & 119.7 & $\mathrm{O} 12-\mathrm{C} 11-\mathrm{C} 10$ & $111.61(16)$ \\
\hline $\mathrm{C} 4-\mathrm{C} 3-\mathrm{C} 2$ & $118.4(2)$ & $\mathrm{O} 12-\mathrm{C} 11-\mathrm{H} 11 \mathrm{~A}$ & 109.3 \\
\hline $\mathrm{C} 4-\mathrm{C} 3-\mathrm{H} 3$ & 120.8 & $\mathrm{C} 10-\mathrm{C} 11-\mathrm{H} 11 \mathrm{~A}$ & 109.3 \\
\hline $\mathrm{C} 2-\mathrm{C} 3-\mathrm{H} 3$ & 120.8 & $\mathrm{O} 12-\mathrm{C} 11-\mathrm{H} 11 \mathrm{~B}$ & 109.3 \\
\hline $\mathrm{C} 5-\mathrm{C} 4-\mathrm{F} 15$ & $118.32(19)$ & $\mathrm{C} 10-\mathrm{C} 11-\mathrm{H} 11 \mathrm{~B}$ & 109.3 \\
\hline $\mathrm{C} 5-\mathrm{C} 4-\mathrm{C} 3$ & $122.83(19)$ & $\mathrm{H} 11 \mathrm{~A}-\mathrm{C} 11-\mathrm{H} 11 \mathrm{~B}$ & 108.0 \\
\hline $\mathrm{F} 15-\mathrm{C} 4-\mathrm{C} 3$ & 118.85 (19) & $\mathrm{C} 13-\mathrm{O} 12-\mathrm{C} 11$ & $110.17(15)$ \\
\hline $\mathrm{C} 4-\mathrm{C} 5-\mathrm{C} 6$ & $118.4(2)$ & $\mathrm{O} 12-\mathrm{C} 13-\mathrm{C} 14$ & $111.15(16)$ \\
\hline $\mathrm{C} 4-\mathrm{C} 5-\mathrm{H} 5$ & 120.8 & $\mathrm{O} 12-\mathrm{C} 13-\mathrm{H} 13 \mathrm{~A}$ & 109.4 \\
\hline $\mathrm{C} 6-\mathrm{C} 5-\mathrm{H} 5$ & 120.8 & $\mathrm{C} 14-\mathrm{C} 13-\mathrm{H} 13 \mathrm{~A}$ & 109.4 \\
\hline $\mathrm{C} 5-\mathrm{C} 6-\mathrm{C} 1$ & $120.6(2)$ & $\mathrm{O} 12-\mathrm{C} 13-\mathrm{H} 13 \mathrm{~B}$ & 109.4 \\
\hline $\mathrm{C} 5-\mathrm{C} 6-\mathrm{H} 6$ & 119.7 & $\mathrm{C} 14-\mathrm{C} 13-\mathrm{H} 13 \mathrm{~B}$ & 109.4 \\
\hline $\mathrm{C} 1-\mathrm{C} 6-\mathrm{H} 6$ & 119.7 & $\mathrm{H} 13 \mathrm{~A}-\mathrm{C} 13-\mathrm{H} 13 \mathrm{~B}$ & 108.0 \\
\hline $\mathrm{O} 8-\mathrm{C} 7-\mathrm{N} 9$ & $122.10(17)$ & N9- $\mathrm{C} 14-\mathrm{C} 13$ & $109.49(16)$ \\
\hline $\mathrm{O} 8-\mathrm{C} 7-\mathrm{C} 1$ & $119.77(18)$ & N9- $\mathrm{C} 14-\mathrm{H} 14 \mathrm{~A}$ & 109.8 \\
\hline $\mathrm{N} 9-\mathrm{C} 7-\mathrm{C} 1$ & $118.13(17)$ & $\mathrm{C} 13-\mathrm{C} 14-\mathrm{H} 14 \mathrm{~A}$ & 109.8 \\
\hline $\mathrm{C} 7-\mathrm{N} 9-\mathrm{C} 14$ & $126.54(16)$ & N9- $\mathrm{C} 14-\mathrm{H} 14 \mathrm{~B}$ & 109.8 \\
\hline $\mathrm{C} 7-\mathrm{N} 9-\mathrm{C} 10$ & $120.76(16)$ & $\mathrm{C} 13-\mathrm{C} 14-\mathrm{H} 14 \mathrm{~B}$ & 109.8 \\
\hline $\mathrm{C} 14-\mathrm{N} 9-\mathrm{C} 10$ & $112.30(15)$ & $\mathrm{H} 14 \mathrm{~A}-\mathrm{C} 14-\mathrm{H} 14 \mathrm{~B}$ & 108.2 \\
\hline $\mathrm{N} 9-\mathrm{C} 10-\mathrm{C} 11$ & $109.50(16)$ & & \\
\hline $\mathrm{C} 6-\mathrm{C} 1-\mathrm{C} 2-\mathrm{C} 3$ & $2.3(3)$ & $\mathrm{C} 2-\mathrm{C} 1-\mathrm{C} 7-\mathrm{N} 9$ & $59.3(3)$ \\
\hline $\mathrm{C} 7-\mathrm{C} 1-\mathrm{C} 2-\mathrm{C} 3$ & $178.2(2)$ & $\mathrm{O} 8-\mathrm{C} 7-\mathrm{N} 9-\mathrm{C} 14$ & $-169.42(18)$ \\
\hline $\mathrm{C} 1-\mathrm{C} 2-\mathrm{C} 3-\mathrm{C} 4$ & $0.0(3)$ & $\mathrm{C} 1-\mathrm{C} 7-\mathrm{N} 9-\mathrm{C} 14$ & $10.6(3)$ \\
\hline $\mathrm{C} 2-\mathrm{C} 3-\mathrm{C} 4-\mathrm{C} 5$ & $-1.4(3)$ & $\mathrm{O} 8-\mathrm{C} 7-\mathrm{N} 9-\mathrm{C} 10$ & $2.7(3)$ \\
\hline
\end{tabular}




\begin{tabular}{|l|l|l|l|}
\hline $\mathrm{C} 2-\mathrm{C} 3-\mathrm{C} 4-\mathrm{F} 15$ & $\mathrm{C} 1-\mathrm{C} 7-\mathrm{N} 9-\mathrm{C} 10$ & $-177.21(17)$ \\
$\mathrm{F} 15-\mathrm{C} 4-\mathrm{C} 5-\mathrm{C} 6$ & $-179.58(19)$ & $\mathrm{C} 7-\mathrm{N} 9-\mathrm{C} 10-\mathrm{C} 11$ & $133.13(19)$ \\
$\mathrm{C} 3-\mathrm{C} 4-\mathrm{C} 5-\mathrm{C} 6$ & $0.5(3)$ & $\mathrm{C} 14-\mathrm{N} 9-\mathrm{C} 10-\mathrm{C} 11$ & $-53.7(2)$ \\
$\mathrm{C} 4-\mathrm{C} 5-\mathrm{C} 6-\mathrm{C} 1$ & $1.9(3)$ & $\mathrm{N} 9-\mathrm{C} 10-\mathrm{C} 11-\mathrm{O} 12$ & $55.6(2)$ \\
$\mathrm{C} 2-\mathrm{C} 1-\mathrm{C} 6-\mathrm{C} 5$ & $-3.2(3)$ & $\mathrm{C} 10-\mathrm{C} 11-\mathrm{O} 12-\mathrm{C} 13$ & $-59.3(2)$ \\
$\mathrm{C} 7-\mathrm{C} 1-\mathrm{C} 6-\mathrm{C} 5$ & $-179.27(19)$ & $\mathrm{C} 11-\mathrm{O} 12-\mathrm{C} 13-\mathrm{C} 14$ & $60.1(2)$ \\
$\mathrm{C} 6-\mathrm{C} 1-\mathrm{C} 7-\mathrm{O} 8$ & $55.3(3)$ & $\mathrm{C} 7-\mathrm{N} 9-\mathrm{C} 14-\mathrm{C} 13$ & $-132.6(2)$ \\
$\mathrm{C} 2-\mathrm{C} 1-\mathrm{C} 7-\mathrm{O} 8$ & $-120.7(2)$ & $\mathrm{C} 10-\mathrm{N} 9-\mathrm{C} 14-\mathrm{C} 13$ & $54.7(2)$ \\
$\mathrm{C} 6-\mathrm{C} 1-\mathrm{C} 7-\mathrm{N} 9$ & $-124.8(2)$ & $\mathrm{O} 12-\mathrm{C} 13-\mathrm{C} 14-\mathrm{N} 9$ & $-57.4(2)$ \\
\hline
\end{tabular}

\section{Computational Methods}

Computational Methods. All of the calculations were performed using Gaussian 09 suite of programs. All of the geometry optimizations were performed at the B3LYP/6-311++G(d,p) level of theory in the gas phase. Extensive studies have showed that this level is accurate in predicting properties and resonance energies of amides. This method was further verified by obtaining good correlations between the calculated structures and X-ray structures in the series. All conformations within $3 \mathrm{kcal} / \mathrm{mol}$ from the lowest energy conformer were explored. The absence of imaginary frequencies was used to characterize the structures as minima on the potential energy surface. All of the optimized geometries were verified as minima (no imaginary frequencies). Electronic and thermal energies were calculated for all structures. Energetic parameters were calculated under standard conditions (298.15 K and $1 \mathrm{~atm})$. For geometry optimizations, we employed the X-ray structures of (4iodophenyl)(morpholino)methanone, (4-bromophenyl)(morpholino)methanone, chlorophenyl)(morpholino)methanone, (4-fluorophenyl)(morpholino)methanone as the starting geometry and performed full optimization for all structures. Optimized amide conformations were used as starting geometries for isodesmic calculations for the aza, keto, and hydrocarbon derivatives. Optimization of all of the protonated structures started with the 
optimized geometries of the amides. Structural representations were generated using CYLview software (Legault, C. Y. CYLview version 1.0 BETA, University of Sherbrooke). All other representations were generated using GaussView (GaussView, version 5, Dennington, R.; Keith, T.; Millam, J. Semichem Inc., Shawnee Mission, KS, 2009).

\section{Full Reference for Gaussian 09}

Gaussian 09, Revision D.01, Frisch, M. J.; Trucks, G. W.; Schlegel, H. B.; Scuseria, G. E.; Robb, M. A.; Cheeseman, J. R.; Scalmani, G.; Barone, V.; Mennucci, B.; Petersson, G. A.; Nakatsuji, H.; Caricato, M.; Li, X.; Hratchian, H. P.; Izmaylov, A. F.; Bloino, J.; Zheng, G.; Sonnenberg, J. L.; Hada, M.; Ehara, M.; Toyota, K.; Fukuda, R.; Hasegawa, J.; Ishida, M.; Nakajima, T.; Honda, Y.; Kitao, O.; Nakai, H.; Vreven, T.; Montgomery, J. A., Jr.; Peralta, J. E.; Ogliaro, F.; Bearpark, M.; Heyd, J. J.; Brothers, E.; Kudin, K. N.; Staroverov, V. N.; Kobayashi, R.; Normand, J.; Raghavachari, K.; Rendell, A.; Burant, J. C.; Iyengar, S. S.; Tomasi, J.; Cossi, M.; Rega, N.; Millam, M. J.; Klene, M.; Knox, J. E.; Cross, J. B.; Bakken, V.; Adamo, C.; Jaramillo, J.; Gomperts, R.; Stratmann, R. E.; Yazyev, O.; Austin, A. J.; Cammi, R.; Pomelli, C.; Ochterski, J. W.; Martin, R. L.; Morokuma, K.; Zakrzewski, V. G.; Voth, G. A.; Salvador, P.; Dannenberg, J. J.; Dapprich, S.; Daniels, A. D.; Farkas, Ö.; Foresman, J. B.; Ortiz, J. V.; Cioslowski, J.; Fox, D. J. Gaussian, Inc., Wallingford CT, 2009.

Cartesian Coordinates with Zero-Point Energies and Thermal Corrections

\begin{tabular}{|c|c|c|c|}
\hline $\begin{array}{l}\text { la } \\
\text { Ene } \\
\text { Sum } \\
\text { Geo }\end{array}$ & $\begin{array}{l}6 \text { au } \\
\text { and thermal }\end{array}$ & rgies: -929 & 38722 au \\
\hline $\mathrm{C}$ & 0.13468700 & 1.62619200 & 0.61496800 \\
\hline $\mathrm{H}$ & -0.21672000 & 2.51880000 & 1.11872200 \\
\hline $\mathrm{C}$ & -0.78026100 & 0.82663500 & -0.07579600 \\
\hline $\mathrm{C}$ & -0.31297300 & -0.29466700 & -0.76764600 \\
\hline $\mathrm{H}$ & -1.00215900 & -0.90471000 & -1.34087600 \\
\hline $\mathrm{C}$ & 1.04092600 & -0.62477700 & -0.75909700 \\
\hline $\mathrm{H}$ & 1.39241600 & -1.48629300 & -1.31245000 \\
\hline $\mathrm{C}$ & 1.93289500 & 0.16744400 & -0.03869400 \\
\hline
\end{tabular}




\begin{tabular}{|c|c|c|c|}
\hline $\mathrm{C}$ & 1.48606800 & 1.29453700 & 0.65045700 \\
\hline $\mathrm{H}$ & 2.18206700 & 1.91505900 & 1.20035900 \\
\hline $\mathrm{N}$ & -3.20346900 & 0.35077800 & 0.06989700 \\
\hline 0 & -2.46674900 & 2.44280800 & -0.44439500 \\
\hline $\mathrm{C}$ & -2.21389400 & 1.28165400 & -0.15022500 \\
\hline $\mathrm{C}$ & -4.59988700 & 0.77684700 & -0.06982600 \\
\hline $\mathrm{H}$ & -4.63764000 & 1.61752900 & -0.76099100 \\
\hline $\mathrm{H}$ & -4.98470900 & 1.11770200 & 0.90063500 \\
\hline $\mathrm{C}$ & -5.43694400 & -0.39229500 & -0.57845300 \\
\hline $\mathrm{H}$ & -6.49638700 & -0.13184500 & -0.57675300 \\
\hline $\mathrm{H}$ & -5.13761400 & -0.64552400 & -1.60616300 \\
\hline 0 & -5.30433700 & -1.54007200 & 0.25634700 \\
\hline $\mathrm{C}$ & -3.95077700 & -1.96453700 & 0.34238900 \\
\hline $\mathrm{H}$ & -3.59326700 & -2.27889100 & -0.64998100 \\
\hline $\mathrm{H}$ & -3.93827500 & -2.82829900 & 1.00933400 \\
\hline $\mathrm{C}$ & -3.05487200 & -0.85651600 & 0.89050800 \\
\hline $\mathrm{H}$ & -3.35778000 & -0.63103600 & 1.92263400 \\
\hline $\mathrm{H}$ & -2.01615700 & -1.17940100 & 0.90715500 \\
\hline$I$ & 3.97980000 & -0.33591600 & -0.00279300 \\
\hline \multicolumn{4}{|c|}{ 1a amine } \\
\hline \multicolumn{4}{|c|}{ Energy: -855.506664 au } \\
\hline \multicolumn{4}{|c|}{$\begin{array}{l}\text { Sum of electronic and thermal Energies: }-85 b .261414 \text { au } \\
\text { Geometry: }\end{array}$} \\
\hline $\mathrm{C}$ & 0.05145500 & 1.57341600 & 0.81232500 \\
\hline $\mathrm{H}$ & -0.30671600 & 2.31401900 & 1.52050000 \\
\hline $\mathrm{C}$ & -0.83328700 & 1.01600700 & -0.11207300 \\
\hline $\mathrm{C}$ & -0.34498500 & 0.05667600 & -1.00559600 \\
\hline $\mathrm{H}$ & -1.02470900 & -0.39463400 & -1.71947500 \\
\hline $\mathrm{C}$ & 0.99085500 & -0.33489200 & -0.98100900 \\
\hline $\mathrm{H}$ & 1.35141200 & -1.07878100 & -1.68036500 \\
\hline $\mathrm{C}$ & 1.85718700 & 0.24062000 & -0.05126700 \\
\hline $\mathrm{C}$ & 1.39460000 & 1.19563400 & 0.84902800 \\
\hline $\mathrm{H}$ & 2.06375000 & 1.63938200 & 1.57529700 \\
\hline $\mathrm{N}$ & -3.22863400 & 0.35819500 & -0.25321300 \\
\hline $\mathrm{C}$ & -2.27948300 & 1.46672000 & -0.17731700 \\
\hline $\mathrm{C}$ & -4.56902700 & 0.80247500 & -0.63879200 \\
\hline $\mathrm{H}$ & -4.50940300 & 1.32164400 & -1.60069200 \\
\hline $\mathrm{H}$ & -4.99107600 & 1.50844200 & 0.10085900 \\
\hline $\mathrm{C}$ & -5.50007100 & -0.39846200 & -0.76723000 \\
\hline $\mathrm{H}$ & -6.52134900 & -0.07396800 & -0.97451100 \\
\hline $\mathrm{H}$ & -5.15881200 & -1.04391700 & -1.58991000 \\
\hline 0 & -5.55184300 & -1.14615600 & 0.44213400 \\
\hline $\mathrm{C}$ & -4.25353700 & -1.58850000 & 0.82457800 \\
\hline $\mathrm{H}$ & -3.86199800 & -2.28716400 & 0.07089400 \\
\hline $\mathrm{H}$ & -4.37521100 & -2.12156000 & 1.76901600 \\
\hline $\mathrm{C}$ & -3.29154100 & -0.41768500 & 0.98824200 \\
\hline $\mathrm{H}$ & -3.62629600 & 0.21249600 & 1.83361800 \\
\hline $\mathrm{H}$ & -2.29408000 & -0.79570500 & 1.22732400 \\
\hline$I$ & 3.88393600 & -0.34743000 & -0.00655400 \\
\hline $\mathrm{H}$ & -2.49234400 & 2.12789100 & 0.68280300 \\
\hline $\mathrm{H}$ & -2.41957700 & 2.07548400 & -1.07790900 \\
\hline \multicolumn{4}{|c|}{ la ketone } \\
\hline \multicolumn{4}{|c|}{$\begin{array}{l}\text { Sum of electronic and thermal Energies: }-913.275713 \text { au } \\
\text { Geometry: }\end{array}$} \\
\hline $\mathrm{C}$ & 0.28280900 & 1.69394500 & 0.38175200 \\
\hline $\mathrm{H}$ & -0.06384700 & 2.67176000 & 0.69316000 \\
\hline $\mathrm{C}$ & -0.66895300 & 0.74590000 & -0.02047500 \\
\hline $\mathrm{C}$ & -0.22242800 & -0.51775900 & -0.42711200 \\
\hline
\end{tabular}




\begin{tabular}{|c|c|c|c|}
\hline $\mathrm{H}$ & -0.92439100 & -1.27626300 & -0.74957500 \\
\hline $\mathrm{C}$ & 1.13497500 & -0.83120000 & -0.43343300 \\
\hline $\mathrm{H}$ & 1.46348600 & -1.81171200 & -0.75338100 \\
\hline $\mathrm{C}$ & 2.06019800 & 0.12808400 & -0.02697200 \\
\hline $\mathrm{C}$ & 1.63893600 & 1.39481300 & 0.38276400 \\
\hline $\mathrm{H}$ & 2.36004300 & 2.13823700 & 0.69787000 \\
\hline 0 & -2.42100400 & 2.29700900 & 0.27946800 \\
\hline $\mathrm{C}$ & -2.11793200 & 1.15116300 & 0.00238400 \\
\hline $\mathrm{C}$ & -4.51473600 & 0.74577100 & -0.74483000 \\
\hline $\mathrm{H}$ & -4.37463700 & 1.26371700 & -1.69915600 \\
\hline $\mathrm{H}$ & -4.83049900 & 1.49557200 & -0.01619700 \\
\hline $\mathrm{C}$ & -5.59908900 & -0.32044600 & -0.89362100 \\
\hline $\mathrm{H}$ & -6.56365300 & 0.13159900 & -1.13017700 \\
\hline $\mathrm{H}$ & -5.34286600 & -1.01840700 & -1.70778200 \\
\hline 0 & -5.79602200 & -1.05434300 & 0.31212400 \\
\hline $\mathrm{C}$ & -4.61423600 & -1.71919200 & 0.73417200 \\
\hline $\mathrm{H}$ & -4.31739900 & -2.46376600 & -0.02324800 \\
\hline $\mathrm{H}$ & -4.87673500 & -2.25461700 & 1.64851400 \\
\hline $\mathrm{C}$ & -3.46359500 & -0.74274900 & 0.98611500 \\
\hline $\mathrm{H}$ & -3.73151800 & -0.07714200 & 1.81297100 \\
\hline $\mathrm{H}$ & -2.57049500 & -1.29904700 & 1.28725600 \\
\hline$I$ & 4.11384200 & -0.33758700 & -0.03169200 \\
\hline $\mathrm{C}$ & -3.19666800 & 0.10772300 & -0.28124600 \\
\hline $\mathrm{H}$ & -2.84056600 & -0.56548800 & -1.07029000 \\
\hline \multicolumn{4}{|c|}{ 1a hydrocarbon } \\
\hline \multicolumn{4}{|c|}{ Energy: -839.479374 au } \\
\hline \multicolumn{4}{|c|}{ Geometry: } \\
\hline $\mathrm{C}$ & -0.08286900 & 0.41182500 & 1.45984400 \\
\hline $\mathrm{H}$ & -0.57102400 & 0.24171400 & 2.41407000 \\
\hline $\mathrm{C}$ & -0.79659200 & 1.00821400 & 0.41624000 \\
\hline $\mathrm{C}$ & -0.12736500 & 1.22069900 & -0.79511600 \\
\hline $\mathrm{H}$ & -0.64951100 & 1.69281700 & -1.62171400 \\
\hline $\mathrm{C}$ & 1.20374000 & 0.84818300 & -0.96717300 \\
\hline $\mathrm{H}$ & 1.69982900 & 1.02757800 & -1.91274200 \\
\hline $\mathrm{C}$ & 1.88844300 & 0.25090800 & 0.09001600 \\
\hline $\mathrm{C}$ & 1.25081000 & 0.03169200 & 1.30785100 \\
\hline $\mathrm{H}$ & 1.78127900 & -0.42657100 & 2.13304600 \\
\hline $\mathrm{C}$ & -2.24833400 & 1.40286300 & 0.57505500 \\
\hline $\mathrm{C}$ & -4.67991300 & 1.01992200 & -0.07109000 \\
\hline $\mathrm{H}$ & -4.73779700 & 1.98502300 & -0.58778400 \\
\hline $\mathrm{H}$ & -4.96381900 & 1.19335000 & 0.97395900 \\
\hline $\mathrm{C}$ & -5.67732600 & 0.04568600 & -0.69921900 \\
\hline $\mathrm{H}$ & -6.70479700 & 0.39090600 & -0.56976700 \\
\hline $\mathrm{H}$ & -5.48380200 & -0.04755000 & -1.78046900 \\
\hline 0 & -5.61488200 & -1.24054300 & -0.09341500 \\
\hline $\mathrm{C}$ & -4.32136400 & -1.82490400 & -0.21268300 \\
\hline $\mathrm{H}$ & -4.08466800 & -1.97763900 & -1.27830300 \\
\hline $\mathrm{H}$ & -4.38987400 & -2.80595700 & 0.26075900 \\
\hline $\mathrm{C}$ & -3.24240800 & -0.96499500 & 0.44598600 \\
\hline $\mathrm{H}$ & -3.43405700 & -0.92354300 & 1.52542100 \\
\hline $\mathrm{H}$ & -2.26496300 & -1.43767200 & 0.30680800 \\
\hline I & 3.90677800 & -0.31531600 & -0.15584800 \\
\hline $\mathrm{H}$ & -2.49938100 & 1.45137700 & 1.64028500 \\
\hline $\mathrm{H}$ & -2.39014300 & 2.41427500 & 0.17685300 \\
\hline $\mathrm{C}$ & -3.24844800 & 0.46040600 & -0.13043100 \\
\hline $\mathrm{H}$ & -2.95770100 & 0.39498600 & -1.18881600 \\
\hline \multicolumn{4}{|l|}{$1 b$} \\
\hline \multicolumn{4}{|c|}{ Energy: -3205.911298 au } \\
\hline
\end{tabular}




\begin{tabular}{|c|c|c|c|}
\hline $\begin{array}{l}\text { Sum } \\
\text { Geom }\end{array}$ & and thermal $\mathrm{E}$ & ergies: -320 & 683290 au \\
\hline $\mathrm{Br}$ & 4.35201100 & -0.46762400 & 0.00506500 \\
\hline $\mathrm{N}$ & -2.61714300 & 0.36714000 & -0.06865400 \\
\hline 0 & -1.82160400 & 2.43853400 & 0.44164000 \\
\hline $\mathrm{C}$ & -1.60165200 & 1.27026700 & 0.14933900 \\
\hline $\mathrm{C}$ & -2.50395600 & -0.84274900 & -0.89124800 \\
\hline $\mathrm{H}$ & -1.47495700 & -1.19525000 & -0.90967900 \\
\hline $\mathrm{H}$ & -2.80134200 & -0.60708500 & -1.92269900 \\
\hline $\mathrm{C}$ & -4.00081200 & 0.83275800 & 0.07254400 \\
\hline $\mathrm{H}$ & -4.37639700 & 1.18576300 & -0.89718500 \\
\hline $\mathrm{H}$ & -4.01416000 & 1.67328700 & 0.76479700 \\
\hline 0 & -4.77144000 & -1.46259500 & -0.25616300 \\
\hline $\mathrm{C}$ & -3.43070100 & -1.92547100 & -0.34387100 \\
\hline $\mathrm{H}$ & -3.44337700 & -2.78831300 & -1.01199500 \\
\hline $\mathrm{H}$ & -3.08155400 & -2.25129000 & 0.64779200 \\
\hline $\mathrm{C}$ & -4.87053200 & -0.31266400 & 0.58029000 \\
\hline $\mathrm{H}$ & -4.57781100 & -0.57575100 & 1.60743100 \\
\hline $\mathrm{H}$ & -5.92208000 & -0.02199200 & 0.57978700 \\
\hline $\mathrm{C}$ & -0.18144000 & 0.77500400 & 0.07509100 \\
\hline $\mathrm{C}$ & 0.75582700 & 1.55085600 & -0.61308400 \\
\hline $\mathrm{H}$ & 0.42953600 & 2.45424000 & -1.11418700 \\
\hline $\mathrm{C}$ & 2.09720300 & 1.18239200 & -0.64923800 \\
\hline $\mathrm{H}$ & 2.81642400 & 1.77970200 & -1.19443100 \\
\hline $\mathrm{C}$ & 2.50467900 & 0.04186500 & 0.03767600 \\
\hline $\mathrm{C}$ & 1.59678700 & -0.72972000 & 0.75630900 \\
\hline $\mathrm{H}$ & 1.93097700 & -1.60069600 & 1.30503600 \\
\hline $\mathrm{C}$ & 0.25326200 & -0.36100300 & 0.76438800 \\
\hline $\mathrm{H}$ & -0.45326200 & -0.95248000 & 1.33563900 \\
\hline $1 b a$ & & & \\
\hline $\begin{array}{l}\text { Ener } \\
\text { Sum } \\
\text { Geom }\end{array}$ & $\begin{array}{l}80 \text { au } \\
\text { and thermal } \mathrm{E}\end{array}$ & ergies: -313 & $606034 \mathrm{au}$ \\
\hline $\mathrm{Br}$ & -4.26988400 & -0.47525500 & -0.01088100 \\
\hline $\mathrm{N}$ & 2.62944900 & 0.36962300 & -0.25284000 \\
\hline $\mathrm{C}$ & 1.65094400 & 1.45223700 & -0.17425700 \\
\hline $\mathrm{C}$ & 2.71140100 & -0.40881200 & 0.98591800 \\
\hline $\mathrm{H}$ & 1.72412500 & -0.81441700 & 1.22205100 \\
\hline $\mathrm{H}$ & 3.02778900 & 0.22707800 & 1.83409000 \\
\hline $\mathrm{C}$ & 3.95802800 & 0.85118600 & -0.63480300 \\
\hline $\mathrm{H}$ & 4.35976700 & 1.56567000 & 0.10796200 \\
\hline $\mathrm{H}$ & 3.88584900 & 1.37195100 & -1.59498700 \\
\hline 0 & 4.99132700 & -1.07396100 & 0.44101400 \\
\hline $\mathrm{C}$ & 3.70487100 & -1.55265400 & 0.81964000 \\
\hline $\mathrm{H}$ & 3.83936800 & -2.08568800 & 1.76234800 \\
\hline $\mathrm{H}$ & 3.33358300 & -2.25888900 & 0.06276100 \\
\hline $\mathrm{C}$ & 4.92125300 & -0.32382600 & -0.76586400 \\
\hline $\mathrm{H}$ & 4.59869000 & -0.97539700 & -1.59126300 \\
\hline $\mathrm{H}$ & 5.93371700 & 0.02876600 & -0.97046100 \\
\hline $\mathrm{C}$ & 0.21757400 & 0.96208500 & -0.11076800 \\
\hline C & -0.67954000 & 1.48499000 & 0.82192900 \\
\hline $\mathrm{H}$ & -0.34010600 & 2.22790000 & 1.53661900 \\
\hline $\mathrm{C}$ & -2.01128000 & 1.06976400 & 0.85883400 \\
\hline $\mathrm{H}$ & -2.69612500 & 1.48219200 & 1.58853900 \\
\hline $\mathrm{C}$ & -2.44248600 & 0.11254600 & -0.05113400 \\
\hline $\mathrm{C}$ & -1.56933300 & -0.43124800 & -0.99016100 \\
\hline $\mathrm{H}$ & -1.91783200 & -1.17722700 & -1.69299900 \\
\hline $\mathrm{C}$ & -0.24565100 & -0.00146500 & -1.01328600 \\
\hline $\mathrm{H}$ & 0.44468000 & -0.42591200 & -1.73322800 \\
\hline $\mathrm{H}$ & 1.84566800 & 2.11632900 & 0.68789000 \\
\hline
\end{tabular}




\begin{tabular}{|c|c|c|c|}
\hline $\mathrm{H}$ & 1.77530500 & 2.06705900 & -1.07299200 \\
\hline \multicolumn{4}{|c|}{ 1b ketone } \\
\hline \multicolumn{4}{|c|}{ Energy: -3189.859682 au } \\
\hline $\begin{array}{l}\text { Sum } \\
\text { Geon }\end{array}$ & \multicolumn{2}{|l|}{ 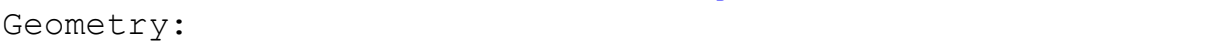 } & 620301 au \\
\hline $\mathrm{Br}$ & -4.50406100 & -0.46382000 & -0.04613000 \\
\hline 0 & 1.76754600 & 2.28427600 & 0.28924700 \\
\hline $\mathrm{C}$ & 1.49432300 & 1.13232600 & 0.00666000 \\
\hline $\mathrm{C}$ & 2.88886800 & -0.72812600 & 0.98615300 \\
\hline $\mathrm{H}$ & 2.01064600 & -1.30830900 & 1.28603100 \\
\hline $\mathrm{H}$ & 3.13926300 & -0.05762700 & 1.81453300 \\
\hline $\mathrm{C}$ & 3.90084400 & 0.79149300 & -0.74108100 \\
\hline $\mathrm{H}$ & 4.19687500 & 1.54736900 & -0.01046500 \\
\hline $\mathrm{H}$ & 3.74731500 & 1.30800900 & -1.69411500 \\
\hline 0 & 5.22860600 & -0.97714200 & 0.31147300 \\
\hline $\mathrm{C}$ & 4.06462900 & -1.67359300 & 0.73190800 \\
\hline $\mathrm{H}$ & 4.34101800 & -2.20413600 & 1.64499400 \\
\hline $\mathrm{H}$ & 3.78727400 & -2.42387100 & -0.02726500 \\
\hline $\mathrm{C}$ & 5.01260500 & -0.24571500 & -0.89247900 \\
\hline $\mathrm{H}$ & 4.77468100 & -0.94805800 & -1.70841200 \\
\hline $\mathrm{H}$ & 5.96502100 & 0.23195800 & -1.12786200 \\
\hline $\mathrm{C}$ & 0.05616700 & 0.69066200 & -0.02115000 \\
\hline $\mathrm{C}$ & -0.92011800 & 1.61486200 & 0.37830200 \\
\hline $\mathrm{H}$ & -0.59866000 & 2.60073700 & 0.69087200 \\
\hline $\mathrm{C}$ & -2.26810000 & 1.28272200 & 0.37528300 \\
\hline $\mathrm{H}$ & -3.01426300 & 2.00229200 & 0.68657000 \\
\hline $\mathrm{C}$ & -2.64891300 & 0.00622900 & -0.03613200 \\
\hline $\mathrm{C}$ & -1.70495300 & -0.93249500 & -0.44010100 \\
\hline $\mathrm{H}$ & -2.01517400 & -1.91885100 & -0.75947500 \\
\hline $\mathrm{C}$ & -0.35657700 & -0.58407100 & -0.42929800 \\
\hline $\mathrm{H}$ & 0.36556400 & -1.32423800 & -0.74925600 \\
\hline $\mathrm{C}$ & 2.59982400 & 0.11799700 & -0.27927800 \\
\hline $\mathrm{H}$ & 2.26178600 & -0.56239400 & -1.07010100 \\
\hline \multicolumn{4}{|c|}{ 1b hydrocarbon } \\
\hline \multicolumn{4}{|c|}{ Energy: -3115.824145 au } \\
\hline \multicolumn{4}{|c|}{ Geometry: } \\
\hline $\mathrm{Br}$ & 4.29773800 & 0.41787000 & -0.23149400 \\
\hline $\mathrm{C}$ & -1.61535700 & -1.35708000 & 0.63278100 \\
\hline $\mathrm{C}$ & -2.66940800 & 0.97686400 & 0.40205600 \\
\hline $\mathrm{H}$ & -1.70564100 & 1.46565700 & 0.22757800 \\
\hline $\mathrm{H}$ & -2.84744800 & 0.98353000 & 1.48460200 \\
\hline $\mathrm{C}$ & -4.06323800 & -1.06515400 & 0.00101600 \\
\hline $\mathrm{H}$ & -4.33026900 & -1.19381600 & 1.05692700 \\
\hline $\mathrm{H}$ & -4.10334700 & -2.05559400 & -0.46712900 \\
\hline 0 & -5.05412200 & 1.16819500 & -0.11927200 \\
\hline $\mathrm{C}$ & -3.77701700 & 1.77716000 & -0.28366300 \\
\hline $\mathrm{H}$ & -3.86424900 & 2.77829400 & 0.14226600 \\
\hline $\mathrm{H}$ & -3.55671600 & 1.88312500 & -1.35843200 \\
\hline $\mathrm{C}$ & -5.09189600 & -0.14734300 & -0.66076400 \\
\hline $\mathrm{H}$ & -4.91367200 & -0.10267400 & -1.74772400 \\
\hline $\mathrm{H}$ & -6.10887600 & -0.51051200 & -0.50113500 \\
\hline $\mathrm{C}$ & -0.17577300 & -0.93537700 & 0.43648700 \\
\hline $\mathrm{C}$ & 0.53430600 & -0.27102700 & 1.44095300 \\
\hline $\mathrm{H}$ & 0.05285000 & -0.06645700 & 2.39161600 \\
\hline $\mathrm{C}$ & 1.85581900 & 0.13427100 & 1.25449400 \\
\hline $\mathrm{H}$ & 2.39027700 & 0.64576600 & 2.04475700 \\
\hline $\mathrm{C}$ & 2.47809200 & -0.13108000 & 0.04051800 \\
\hline $\mathrm{C}$ & 1.80311500 & -0.79483800 & -0.97958000 \\
\hline
\end{tabular}




\begin{tabular}{|c|c|c|c|}
\hline $\mathrm{H}$ & 2.29908900 & -1.00478100 & -1.91860100 \\
\hline $\mathrm{C}$ & 0.48422200 & -1.19072400 & -0.77177300 \\
\hline $\mathrm{H}$ & -0.03552300 & -1.71552000 & -1.56730100 \\
\hline $\mathrm{H}$ & -1.85267000 & -1.35985100 & 1.70223600 \\
\hline $\mathrm{H}$ & -1.73668000 & -2.38985600 & 0.28601300 \\
\hline $\mathrm{C}$ & -2.64691700 & -0.47472200 & -0.10432400 \\
\hline $\mathrm{H}$ & -2.37067300 & -0.45403600 & -1.16841900 \\
\hline \multicolumn{4}{|l|}{$1 c$} \\
\hline \multicolumn{4}{|c|}{ Energy: -1091.991668 au } \\
\hline \multicolumn{4}{|c|}{ Geometry: } \\
\hline $\mathrm{N}$ & 1.91335500 & 0.38821300 & 0.06712000 \\
\hline O & 1.01175300 & 2.41727800 & -0.43645100 \\
\hline $\mathrm{C}$ & 0.85209100 & 1.23833600 & -0.14770400 \\
\hline $\mathrm{C}$ & 1.86428600 & -0.82509000 & 0.89106400 \\
\hline $\mathrm{H}$ & 0.85522300 & -1.23106200 & 0.91055400 \\
\hline $\mathrm{H}$ & 2.14942200 & -0.57304000 & 1.92213400 \\
\hline $\mathrm{C}$ & 3.27065800 & 0.92575600 & -0.07451500 \\
\hline $\mathrm{H}$ & 3.62735800 & 1.29901300 & 0.89478600 \\
\hline $\mathrm{H}$ & 3.23988300 & 1.76521400 & -0.76751400 \\
\hline 0 & 4.16099100 & -1.32565800 & 0.25603200 \\
\hline $\mathrm{C}$ & 2.84642400 & -1.85821100 & 0.34448300 \\
\hline $\mathrm{H}$ & 2.90452500 & -2.71854700 & 1.01344400 \\
\hline $\mathrm{H}$ & 2.51471900 & -2.20289300 & -0.64679600 \\
\hline $\mathrm{C}$ & 4.19930300 & -0.17280500 & -0.58141400 \\
\hline $\mathrm{H}$ & 3.92060400 & -0.45185500 & -1.60824500 \\
\hline $\mathrm{H}$ & 5.23412200 & 0.17273000 & -0.58142000 \\
\hline $\mathrm{C}$ & -0.54032900 & 0.67019400 & -0.07464100 \\
\hline $\mathrm{C}$ & -1.51787200 & 1.39861600 & 0.60971600 \\
\hline $\mathrm{H}$ & -1.23920200 & 2.31900700 & 1.10841700 \\
\hline $\mathrm{C}$ & -2.83779600 & 0.96137100 & 0.64477600 \\
\hline $\mathrm{H}$ & -3.59153500 & 1.51918200 & 1.18549500 \\
\hline $\mathrm{C}$ & -3.18485000 & -0.20057200 & -0.03912100 \\
\hline $\mathrm{C}$ & -2.23695500 & -0.92609700 & -0.75383200 \\
\hline $\mathrm{H}$ & -2.52962000 & -1.81421900 & -1.29909000 \\
\hline $\mathrm{C}$ & -0.91476100 & -0.48885600 & -0.76123500 \\
\hline $\mathrm{H}$ & -0.17765600 & -1.04438100 & -1.32975100 \\
\hline $\mathrm{Cl}$ & -4.85202800 & -0.75443800 & -0.01023300 \\
\hline \multicolumn{4}{|c|}{ 1c amine } \\
\hline \multicolumn{4}{|c|}{ Energy: -1017.931705 au } \\
\hline \multicolumn{4}{|c|}{ Geometry: } \\
\hline $\mathrm{N}$ & 1.90440600 & 0.37920500 & -0.25143200 \\
\hline $\mathrm{C}$ & 0.87409900 & 1.41251500 & -0.16916900 \\
\hline $\mathrm{C}$ & 2.02339000 & -0.39975100 & 0.98397700 \\
\hline $\mathrm{H}$ & 1.05697200 & -0.85437400 & 1.21708800 \\
\hline $\mathrm{H}$ & 2.30721100 & 0.24718200 & 1.83534500 \\
\hline $\mathrm{C}$ & 3.20800900 & 0.92709100 & -0.62996600 \\
\hline $\mathrm{H}$ & 3.57347500 & 1.65743400 & 0.11613600 \\
\hline $\mathrm{H}$ & 3.11109400 & 1.44768000 & -1.58807000 \\
\hline 0 & 4.33387100 & -0.94924200 & 0.43911400 \\
\hline $\mathrm{C}$ & 3.07220800 & -1.49247100 & 0.81400400 \\
\hline $\mathrm{H}$ & 3.23183000 & -2.02236700 & 1.75456700 \\
\hline $\mathrm{H}$ & 2.73708500 & -2.21285500 & 0.05357100 \\
\hline $\mathrm{C}$ & 4.22809100 & -0.19845900 & -0.76474400 \\
\hline $\mathrm{H}$ & 3.93884100 & -0.86176000 & -1.59314700 \\
\hline $\mathrm{H}$ & 5.22215900 & 0.20441600 & -0.96672000 \\
\hline $\mathrm{C}$ & -0.53381300 & 0.85323600 & -0.10866900 \\
\hline $\mathrm{C}$ & -1.45551600 & 1.32723400 & 0.82631900 \\
\hline
\end{tabular}




\begin{tabular}{|lrrr|}
\hline $\mathrm{H}$ & -1.15235300 & 2.08181900 & 1.54494400 \\
$\mathrm{C}$ & -2.76509700 & 0.84799300 & 0.85998600 \\
$\mathrm{H}$ & -3.47281800 & 1.22007000 & 1.58981200 \\
$\mathrm{C}$ & -3.14979900 & -0.12388300 & -0.05501500 \\
$\mathrm{C}$ & -2.25095400 & -0.61952500 & -0.99620500 \\
$\mathrm{H}$ & -2.56744900 & -1.37775900 & -1.70134900 \\
$\mathrm{C}$ & -0.94999600 & -0.12664300 & -1.01672800 \\
$\mathrm{H}$ & -0.23980500 & -0.51318100 & -1.73858800 \\
$\mathrm{Cl}$ & -4.79748500 & -0.74346200 & -0.02177000 \\
$\mathrm{H}$ & 1.03601200 & 2.08150000 & 0.69598700 \\
$\mathrm{H}$ & 0.96944700 & 2.03653300 & -1.06511300
\end{tabular}

\section{1c ketone}

Energy: -1075.940131 au

Sum of electronic and thermal Energies: -1075.700374 au Geometry:

$\begin{array}{lrrr}\mathrm{O} & 0.95508400 & 2.25097100 & 0.30276800 \\ \mathrm{C} & 0.73532000 & 1.08977500 & 0.01111300 \\ \mathrm{C} & 2.21206200 & -0.71016200 & 0.98367800 \\ \mathrm{H} & 1.36092500 & -1.33222400 & 1.27769300 \\ \mathrm{H} & 2.42889000 & -0.03368800 & 1.81665600 \\ \mathrm{C} & 3.15676200 & 0.86473400 & -0.73213600 \\ \mathrm{H} & 3.41557900 & 1.62935800 & 0.00347700 \\ \mathrm{H} & 2.98175500 & 1.37900300 & -1.68268800 \\ \mathrm{O} & 4.56257100 & -0.84630100 & 0.31411800 \\ \mathrm{C} & 3.43108800 & -1.59847400 & 0.72719800 \\ \mathrm{H} & 3.72951200 & -2.12117000 & 1.63787600 \\ \mathrm{H} & 3.19076800 & -2.35616100 & -0.03722400 \\ \mathrm{C} & 4.31592400 & -0.11878200 & -0.88629400 \\ \mathrm{H} & 4.11302900 & -0.82674000 & -1.70683000 \\ \mathrm{H} & 5.24571400 & 0.40398200 & -1.11626800 \\ \mathrm{C} & -0.68061200 & 0.58330100 & -0.02548100 \\ \mathrm{C} & -1.70028000 & 1.46077800 & 0.37175700 \\ \mathrm{H} & -1.42539700 & 2.45897500 & 0.68931100 \\ \mathrm{C} & -3.03098700 & 1.06757900 & 0.35984400 \\ \mathrm{H} & -3.81440800 & 1.74792000 & 0.66838100 \\ \mathrm{C} & -3.35133300 & -0.22331600 & -0.05767900 \\ \mathrm{C} & -2.36338400 & -1.11648800 & -0.45939600 \\ \mathrm{H} & -2.63159100 & -2.11394000 & -0.78285300 \\ \mathrm{C} & -1.03288600 & -0.70757100 & -0.44008300 \\ \mathrm{H} & -0.27592000 & -1.41270500 & -0.75839600 \\ \mathrm{Cl} & -5.03036400 & -0.73063000 & -0.07765600 \\ \mathrm{C} & 1.88718600 & 0.12902400 & -0.27765000 \\ \mathrm{H} & 1.58294200 & -0.56165400 & -1.07328500 \\ & & \end{array}$

\section{1c hydrocarbon}

Energy: -1001.904400 au

Sum of electronic and thermal Energies: -1001.646755 au Geometry:

$\begin{array}{lrrr}\mathrm{C} & 0.83418600 & 1.26221600 & 0.72168400 \\ \mathrm{C} & 1.98834800 & -0.99953100 & 0.31698400 \\ \mathrm{H} & 1.04985000 & -1.50797500 & 0.07425100 \\ \mathrm{H} & 2.14062300 & -1.09488900 & 1.39929000 \\ \mathrm{C} & 3.30615400 & 1.12253000 & 0.13339500 \\ \mathrm{H} & 3.54250900 & 1.16800900 & 1.20338500 \\ \mathrm{H} & 3.31664400 & 2.15115600 & -0.24550600 \\ \mathrm{O} & 4.39061800 & -1.05135500 & -0.15385900 \\ \mathrm{C} & 3.14384700 & -1.69256200 & -0.40544800 \\ \mathrm{H} & 3.26202300 & -2.72325400 & -0.06630400 \\ \mathrm{H} & 2.95368200 & -1.71164800 & -1.49097400 \\ \mathrm{C} & 4.38713300 & 0.30727300 & -0.57745900\end{array}$




\begin{tabular}{|c|c|c|c|}
\hline $\mathrm{H}$ & 4.23681700 & 0.35168900 & -1.66864700 \\
\hline $\mathrm{H}$ & 5.38425000 & 0.69418700 & -0.35908800 \\
\hline $\mathrm{C}$ & -0.58192500 & 0.80207600 & 0.45355800 \\
\hline $\mathrm{C}$ & -1.28568800 & 0.02633800 & 1.37969600 \\
\hline $\mathrm{H}$ & -0.81686500 & -0.23963700 & 2.32134400 \\
\hline $\mathrm{C}$ & -2.58427500 & -0.41319500 & 1.12584400 \\
\hline $\mathrm{H}$ & -3.11815800 & -1.01162300 & 1.85316900 \\
\hline $\mathrm{C}$ & -3.19054000 & -0.06995600 & -0.07645000 \\
\hline $\mathrm{C}$ & -2.52139100 & 0.70502800 & -1.01871800 \\
\hline $\mathrm{H}$ & -3.00912200 & 0.97241000 & -1.94759800 \\
\hline $\mathrm{C}$ & -1.22547100 & 1.13368600 & -0.74512200 \\
\hline $\mathrm{H}$ & -0.71060500 & 1.74489600 & -1.47968400 \\
\hline $\mathrm{Cl}$ & -4.83079400 & -0.61641200 & -0.41022000 \\
\hline $\mathrm{H}$ & 1.04752500 & 1.18178900 & 1.79319800 \\
\hline $\mathrm{H}$ & 0.91992600 & 2.32522800 & 0.46812200 \\
\hline $\mathrm{C}$ & 1.91817000 & 0.48909400 & -0.06131200 \\
\hline $\mathrm{H}$ & 1.66817100 & 0.55152900 & -1.13027400 \\
\hline \multicolumn{4}{|c|}{$1 d$} \\
\hline \multicolumn{4}{|c|}{ Energy: -731.637720 au } \\
\hline \multicolumn{4}{|c|}{$\begin{array}{l}\text { Sum of electronic and thermal thergies: }-131.4083 / 9 \text { au } \\
\text { Geometry: }\end{array}$} \\
\hline $\mathrm{C}$ & 0.96197800 & 0.54660100 & -0.07091300 \\
\hline $\mathrm{C}$ & 1.26200100 & -0.64034000 & -0.74787300 \\
\hline $\mathrm{H}$ & 0.48866200 & -1.15512800 & -1.30613500 \\
\hline $\mathrm{C}$ & 2.55615700 & -1.15539800 & -0.74394100 \\
\hline $\mathrm{H}$ & 2.80938300 & -2.06332000 & -1.27683600 \\
\hline $\mathrm{C}$ & 3.53625600 & -0.47099100 & -0.04227200 \\
\hline $\mathrm{C}$ & 3.27751600 & 0.71260100 & 0.63280300 \\
\hline $\mathrm{H}$ & 4.07769900 & 1.21932600 & 1.15752400 \\
\hline $\mathrm{C}$ & 1.98478600 & 1.22511800 & 0.60045900 \\
\hline $\mathrm{H}$ & 1.76095100 & 2.16536500 & 1.08926300 \\
\hline 0 & -0.48434200 & 2.38537800 & -0.42097300 \\
\hline $\mathrm{C}$ & -0.39338300 & 1.19659300 & -0.14258900 \\
\hline $\mathrm{N}$ & -1.50486500 & 0.40837300 & 0.06096700 \\
\hline $\mathrm{C}$ & -2.82620000 & 1.02801000 & -0.08500300 \\
\hline $\mathrm{H}$ & -2.74326200 & 1.86044300 & -0.78220200 \\
\hline $\mathrm{H}$ & -3.16058400 & 1.42807000 & 0.88164300 \\
\hline $\mathrm{C}$ & -3.82040300 & -0.01339600 & -0.58797300 \\
\hline $\mathrm{H}$ & -4.83176500 & 0.39576100 & -0.59191200 \\
\hline $\mathrm{H}$ & -3.55783400 & -0.31469600 & -1.61278200 \\
\hline 0 & -3.85551900 & -1.16213900 & 0.25532700 \\
\hline $\mathrm{C}$ & -2.57644600 & -1.77414600 & 0.35011600 \\
\hline $\mathrm{H}$ & -2.26476300 & -2.14484500 & -0.63829800 \\
\hline $\mathrm{H}$ & -2.68891000 & -2.62511800 & 1.02414600 \\
\hline $\mathrm{C}$ & -1.53343000 & -0.80040800 & 0.89270100 \\
\hline $\mathrm{H}$ & -1.80496700 & -0.52474300 & 1.92151700 \\
\hline $\mathrm{H}$ & -0.55167700 & -1.26822800 & 0.91773200 \\
\hline $\mathrm{F}$ & 4.79411300 & -0.97143200 & -0.02426100 \\
\hline \multicolumn{4}{|c|}{ 1d amine } \\
\hline \multicolumn{4}{|c|}{$\begin{array}{l}\text { Sum of electronic and thermal Energies: } \mathbf{- 6 5 7 . 3 3 0 6 7 6} \text { au } \\
\text { Geometry: }\end{array}$} \\
\hline $\mathrm{C}$ & 0.97692100 & 0.73545300 & -0.10210700 \\
\hline $\mathrm{C}$ & 1.34783400 & -0.23660200 & -1.03887500 \\
\hline $\mathrm{H}$ & 0.62297400 & -0.56170500 & -1.77612200 \\
\hline $\mathrm{C}$ & 2.62126600 & -0.79798000 & -1.02607600 \\
\hline $\mathrm{H}$ & 2.91893300 & -1.55077600 & -1.74565000 \\
\hline $\mathrm{C}$ & 3.52394600 & -0.37164500 & -0.06264700 \\
\hline $\mathrm{C}$ & 3.19724900 & 0.58548000 & 0.88200700 \\
\hline
\end{tabular}




\begin{tabular}{|c|c|c|c|}
\hline $\mathrm{H}$ & 3.92896000 & 0.88682000 & 1.62122800 \\
\hline $\mathrm{C}$ & 1.91377900 & 1.13260600 & 0.85418500 \\
\hline $\mathrm{H}$ & 1.64237400 & 1.87971700 & 1.59279600 \\
\hline $\mathrm{C}$ & -0.40007100 & 1.36803000 & -0.15449000 \\
\hline $\mathrm{N}$ & -1.48331400 & 0.39053300 & -0.24750600 \\
\hline $\mathrm{C}$ & -2.75787200 & 1.01108900 & -0.61185100 \\
\hline $\mathrm{H}$ & -2.63734700 & 1.54144000 & -1.56191000 \\
\hline $\mathrm{H}$ & -3.08249100 & 1.74736700 & 0.14730000 \\
\hline $\mathrm{C}$ & -3.83551200 & -0.05763600 & -0.76049000 \\
\hline $\mathrm{H}$ & -4.80805200 & 0.39941500 & -0.95176300 \\
\hline $\mathrm{H}$ & -3.58388300 & -0.72147000 & -1.60068400 \\
\hline 0 & -3.97628700 & -0.82160500 & 0.43151900 \\
\hline $\mathrm{C}$ & -2.74296000 & -1.43452200 & 0.79331800 \\
\hline $\mathrm{H}$ & -2.44740100 & -2.15913400 & 0.02059500 \\
\hline $\mathrm{H}$ & -2.92699900 & -1.97018200 & 1.72615800 \\
\hline $\mathrm{C}$ & -1.63881500 & -0.40000400 & 0.97621400 \\
\hline $\mathrm{H}$ & -1.88666200 & 0.24747900 & 1.83843400 \\
\hline $\mathrm{H}$ & -0.69637700 & -0.90710000 & 1.19887200 \\
\hline $\mathrm{F}$ & 4.76746400 & -0.91545300 & -0.04457500 \\
\hline $\mathrm{H}$ & -0.52662500 & 2.03338400 & 0.71939400 \\
\hline $\mathrm{H}$ & -0.46566900 & 2.00730000 & -1.04222000 \\
\hline \multicolumn{4}{|c|}{ 1d ketone } \\
\hline \multicolumn{4}{|c|}{ Energy: -715.586408 au } \\
\hline \multicolumn{3}{|c|}{ Geometry: } & 45686 au \\
\hline $\mathrm{C}$ & 1.10488900 & 0.46521100 & -0.03218800 \\
\hline $\mathrm{C}$ & 1.38973800 & -0.84069500 & -0.45463100 \\
\hline $\mathrm{H}$ & 0.59567800 & -1.50463700 & -0.77049900 \\
\hline $\mathrm{C}$ & 2.69809200 & -1.31532700 & -0.48337600 \\
\hline $\mathrm{H}$ & 2.93245400 & -2.32070200 & -0.80965700 \\
\hline $\mathrm{C}$ & 3.71705600 & -0.46532400 & -0.08326900 \\
\hline $\mathrm{C}$ & 3.47860900 & 0.83506100 & 0.34166500 \\
\hline $\mathrm{H}$ & 4.30765800 & 1.46242200 & 0.64447000 \\
\hline $\mathrm{C}$ & 2.16852000 & 1.29178700 & 0.36296700 \\
\hline $\mathrm{H}$ & 1.94181700 & 2.30013200 & 0.68594200 \\
\hline 0 & -0.44618000 & 2.20698900 & 0.32186400 \\
\hline $\mathrm{C}$ & -0.28319400 & 1.03943500 & 0.01730500 \\
\hline $\mathrm{C}$ & -2.71395000 & 0.94093500 & -0.72368300 \\
\hline $\mathrm{H}$ & -2.51428000 & 1.44999000 & -1.67220300 \\
\hline $\mathrm{H}$ & -2.93210300 & 1.71435800 & 0.01587800 \\
\hline $\mathrm{C}$ & -3.92225300 & 0.01888900 & -0.88044400 \\
\hline $\mathrm{H}$ & -4.82441600 & 0.58971800 & -1.10620500 \\
\hline $\mathrm{H}$ & -3.75726000 & -0.69460900 & -1.70469900 \\
\hline 0 & -4.20399000 & -0.70098000 & 0.31686100 \\
\hline $\mathrm{C}$ & -3.11178300 & -1.51215900 & 0.72404600 \\
\hline $\mathrm{H}$ & -2.91187800 & -2.27720200 & -0.04470000 \\
\hline $\mathrm{H}$ & -3.43518000 & -2.02362600 & 1.63259300 \\
\hline $\mathrm{C}$ & -1.84852500 & -0.68872900 & 0.98264600 \\
\hline $\mathrm{H}$ & -2.02898900 & -0.00641100 & 1.81950600 \\
\hline $\mathrm{H}$ & -1.02986400 & -1.35511300 & 1.27189200 \\
\hline $\mathrm{F}$ & 4.98967100 & -0.91897000 & -0.10833800 \\
\hline $\mathrm{C}$ & -1.48281900 & 0.13926600 & -0.27478900 \\
\hline $\mathrm{H}$ & -1.21558700 & -0.56176100 & -1.07457900 \\
\hline \multicolumn{4}{|c|}{ 1d hydrocarbon } \\
\hline \multicolumn{4}{|c|}{ Energy: -641.549964 au } \\
\hline \multicolumn{4}{|c|}{$\begin{array}{l}\text { Sum of electronic and thermal Energies: }-641.291370 \text { au } \\
\text { Geometry: }\end{array}$} \\
\hline $\mathrm{C}$ & 1.03023200 & 0.65081900 & 0.46512000 \\
\hline $\mathrm{C}$ & 1.65560300 & 1.09555300 & -0.70690400 \\
\hline
\end{tabular}




\begin{tabular}{|c|c|c|c|}
\hline $\mathrm{H}$ & 1.14721500 & 1.80858100 & -1.34804000 \\
\hline $\mathrm{C}$ & 2.92478400 & 0.65067600 & -1.06872300 \\
\hline $\mathrm{H}$ & 3.41331700 & 0.99613300 & -1.97146500 \\
\hline $\mathrm{C}$ & 3.57010200 & -0.25176100 & -0.23770000 \\
\hline $\mathrm{C}$ & 2.99429700 & -0.71510200 & 0.93276100 \\
\hline $\mathrm{H}$ & 3.53349500 & -1.41514000 & 1.55893900 \\
\hline $\mathrm{C}$ & 1.72230400 & -0.25602600 & 1.27451200 \\
\hline $\mathrm{H}$ & 1.26482100 & -0.61000200 & 2.19228900 \\
\hline $\mathrm{C}$ & -0.35795200 & 1.13267400 & 0.82812800 \\
\hline $\mathrm{C}$ & -2.84945200 & 1.15848400 & 0.30836700 \\
\hline $\mathrm{H}$ & -2.82691300 & 2.22391200 & 0.05114600 \\
\hline $\mathrm{H}$ & -3.05414700 & 1.08824200 & 1.38354600 \\
\hline $\mathrm{C}$ & -3.98322300 & 0.47424100 & -0.45636300 \\
\hline $\mathrm{H}$ & -4.95674000 & 0.87135400 & -0.16270000 \\
\hline $\mathrm{H}$ & -3.86140900 & 0.63969200 & -1.53945500 \\
\hline 0 & -4.03248000 & -0.92343600 & -0.19258200 \\
\hline $\mathrm{C}$ & -2.82109500 & -1.57865900 & -0.55620300 \\
\hline $\mathrm{H}$ & -2.66172600 & -1.47849800 & -1.64226100 \\
\hline $\mathrm{H}$ & -2.97344200 & -2.63651100 & -0.33459200 \\
\hline $\mathrm{C}$ & -1.61804700 & -1.01977800 & 0.20376000 \\
\hline $\mathrm{H}$ & -1.74445700 & -1.23453100 & 1.27222800 \\
\hline $\mathrm{H}$ & -0.70876100 & -1.53235300 & -0.12585400 \\
\hline $\mathrm{F}$ & 4.80869700 & -0.68967100 & -0.58054000 \\
\hline $\mathrm{H}$ & -0.54558800 & 0.93788800 & 1.88979300 \\
\hline $\mathrm{H}$ & -0.40544300 & 2.22050600 & 0.70128100 \\
\hline $\mathrm{C}$ & -1.49523600 & 0.49888200 & -0.00250500 \\
\hline $\mathrm{H}$ & -1.27256600 & 0.67523600 & -1.06483400 \\
\hline \multicolumn{4}{|c|}{ le } \\
\hline \multicolumn{4}{|c|}{ Energy: -632.369172 au } \\
\hline \multicolumn{4}{|c|}{ 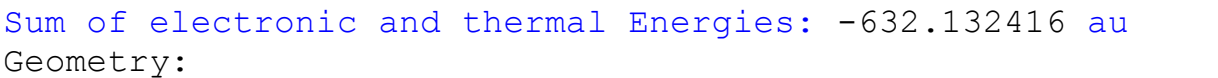 } \\
\hline $\mathrm{C}$ & 1.37057500 & 0.36835600 & -0.08221800 \\
\hline $\mathrm{C}$ & 1.58205700 & -0.82136500 & -0.78728900 \\
\hline $\mathrm{H}$ & 0.77532200 & -1.25480800 & -1.36799600 \\
\hline $\mathrm{C}$ & 2.83349700 & -1.43418100 & -0.77370100 \\
\hline $\mathrm{H}$ & 2.99291300 & -2.34680700 & -1.33699000 \\
\hline $\mathrm{C}$ & 3.87896200 & -0.87163900 & -0.04319600 \\
\hline $\mathrm{C}$ & 3.67500800 & 0.31757800 & 0.65647700 \\
\hline $\mathrm{H}$ & 4.48834300 & 0.76412200 & 1.21726000 \\
\hline $\mathrm{C}$ & 2.43162800 & 0.94269600 & 0.62485300 \\
\hline $\mathrm{H}$ & 2.27462500 & 1.88415800 & 1.13763700 \\
\hline 0 & 0.06790000 & 2.31127700 & -0.44763700 \\
\hline $\mathrm{C}$ & 0.06923900 & 1.12258300 & -0.15398300 \\
\hline $\mathrm{N}$ & -1.09796300 & 0.42638900 & 0.07037400 \\
\hline $\mathrm{C}$ & -2.37014100 & 1.14311800 & -0.06019300 \\
\hline $\mathrm{H}$ & -2.22910300 & 1.97563300 & -0.74792700 \\
\hline $\mathrm{H}$ & -2.66903800 & 1.55428800 & 0.91348700 \\
\hline $\mathrm{C}$ & -3.44156400 & 0.18415200 & -0.56870200 \\
\hline $\mathrm{H}$ & -4.42036000 & 0.66614000 & -0.56015700 \\
\hline $\mathrm{H}$ & -3.20822000 & -0.12237400 & -1.59903300 \\
\hline 0 & -3.55539700 & -0.96978500 & 0.26061300 \\
\hline $\mathrm{C}$ & -2.32363100 & -1.67518800 & 0.33836800 \\
\hline $\mathrm{H}$ & -2.04621700 & -2.05419300 & -0.65699600 \\
\hline $\mathrm{H}$ & -2.49444000 & -2.52463100 & 1.00205300 \\
\hline $\mathrm{C}$ & -1.20820300 & -0.78805900 & 0.88549100 \\
\hline $\mathrm{H}$ & -1.45071700 & -0.50844000 & 1.92051600 \\
\hline $\mathrm{H}$ & -0.26267400 & -1.32592400 & 0.89385200 \\
\hline $\mathrm{H}$ & 4.85072900 & -1.35213300 & -0.02756100 \\
\hline
\end{tabular}




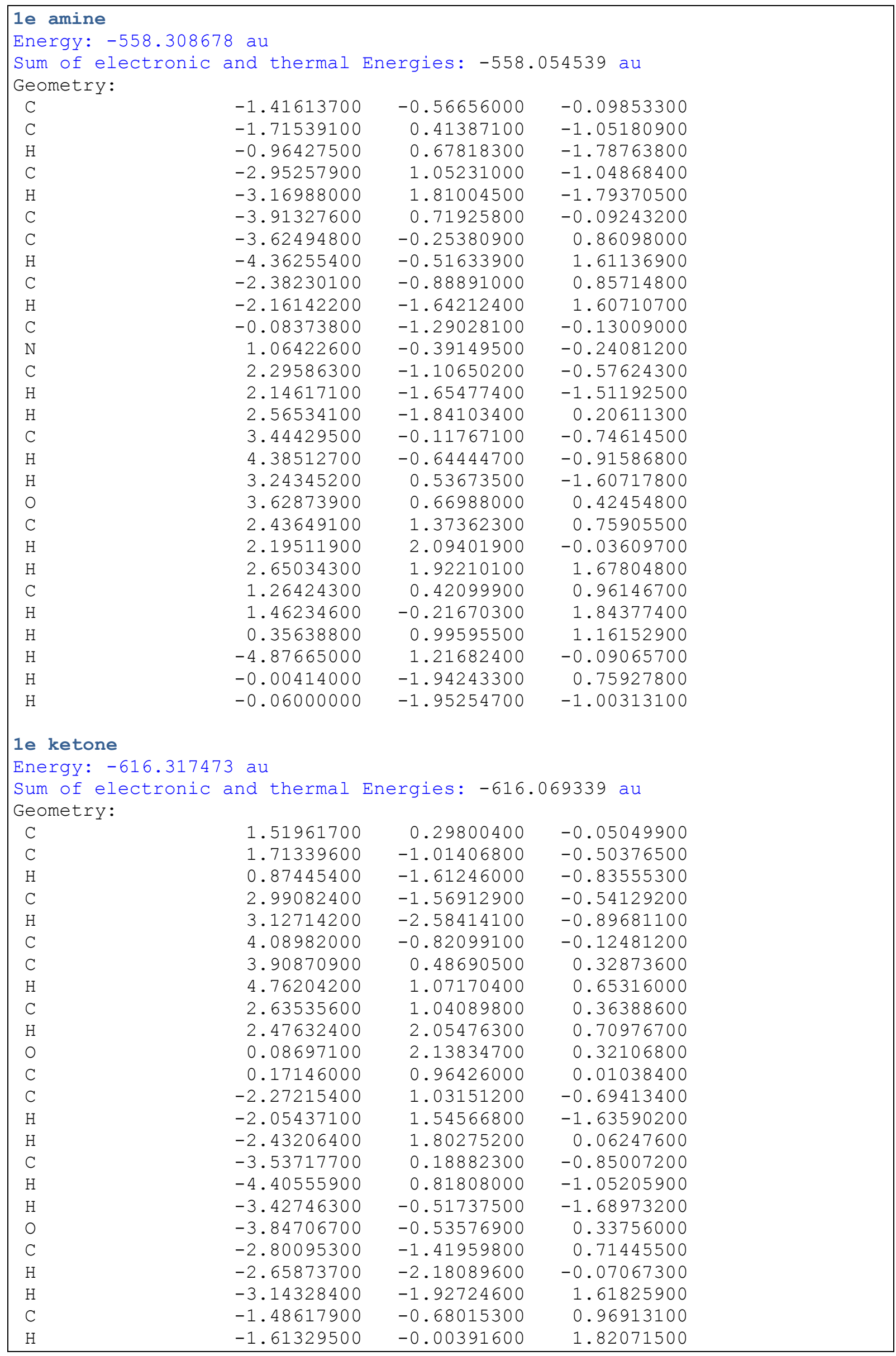




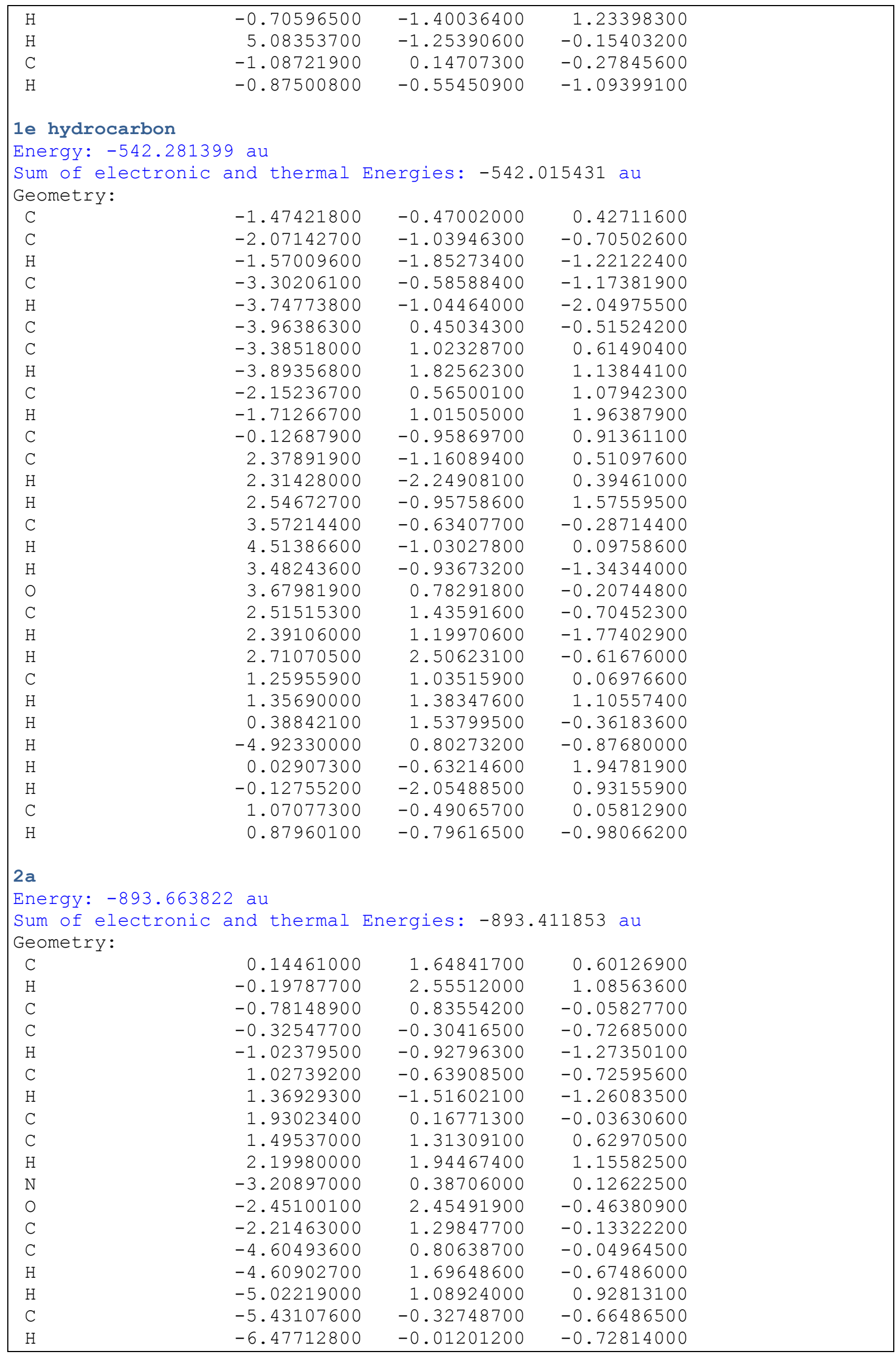




\begin{tabular}{|lrrr}
\hline $\mathrm{H}$ & -5.08564000 & -0.50006500 & -1.69058400 \\
$\mathrm{C}$ & -3.83182400 & -1.99490800 & 0.35403800 \\
$\mathrm{H}$ & -3.38401900 & -2.27882200 & -0.60541300 \\
$\mathrm{H}$ & -3.74036700 & -2.85818000 & 1.02075300 \\
$\mathrm{C}$ & -3.04733500 & -0.81993500 & 0.94598400 \\
$\mathrm{H}$ & -3.42533900 & -0.60561600 & 1.95742300 \\
$\mathrm{H}$ & -1.99125400 & -1.05876800 & 1.04264400 \\
$\mathrm{I}$ & 3.97647500 & -0.34201100 & -0.01172700 \\
$\mathrm{C}$ & -5.30616200 & -1.62221100 & 0.14878100 \\
$\mathrm{H}$ & -5.84129900 & -2.43876900 & -0.34482900 \\
$\mathrm{H}$ & -5.78158400 & -1.48247400 & 1.12826000
\end{tabular}

\section{$2 a$ amine}

Energy: -819.602728 au

Sum of electronic and thermal Energies: -819.333441 au Geometry:

$\begin{array}{lrrr}\mathrm{C} & 0.07550100 & 1.59971700 & 0.80433000 \\ \mathrm{H} & -0.27080800 & 2.36033700 & 1.49713100 \\ \mathrm{C} & -0.82423900 & 1.01961200 & -0.09137200 \\ \mathrm{C} & -0.35100500 & 0.03445100 & -0.96445600 \\ \mathrm{H} & -1.04337400 & -0.43442200 & -1.65437300 \\ \mathrm{C} & 0.98416300 & -0.36037000 & -0.94801500 \\ \mathrm{H} & 1.33260700 & -1.12498800 & -1.63102000 \\ \mathrm{C} & 1.86515600 & 0.23842800 & -0.04752200 \\ \mathrm{C} & 1.41811700 & 1.21951300 & 0.83236700 \\ \mathrm{H} & 2.09877900 & 1.68110000 & 1.53653000 \\ \mathrm{~N} & -3.22669800 & 0.37727600 & -0.23550300 \\ \mathrm{C} & -2.26927700 & 1.47699300 & -0.15131800 \\ \mathrm{C} & -4.55245800 & 0.83782400 & -0.65579600 \\ \mathrm{H} & -4.43910200 & 1.40632500 & -1.58367100 \\ \mathrm{H} & -4.98900400 & 1.52772700 & 0.09470300 \\ \mathrm{C} & -5.50540000 & -0.33944500 & -0.87362900 \\ \mathrm{H} & -6.49164500 & 0.04137900 & -1.15794200 \\ \mathrm{H} & -5.13553300 & -0.94134400 & -1.71115400 \\ \mathrm{C} & -4.20170600 & -1.61671000 & 0.85789100 \\ \mathrm{H} & -3.75024600 & -2.30128000 & 0.13125900 \\ \mathrm{H} & -4.25481300 & -2.14796500 & 1.81355000 \\ \mathrm{C} & -3.29637600 & -0.39293300 & 1.01051700 \\ \mathrm{H} & -3.67232600 & 0.24315700 & 1.83763800 \\ \mathrm{H} & -2.28504400 & -0.70423200 & 1.28189900 \\ \mathrm{I} & 3.89138200 & -0.35436000 & -0.01498100 \\ \mathrm{C} & -5.60199600 & -1.21005300 & 0.38476700 \\ \mathrm{H} & -6.21731600 & -2.09477200 & 0.19419200 \\ \mathrm{H} & -6.10321300 & -0.64331500 & 1.18010600 \\ \mathrm{H} & -2.47565900 & 2.13517200 & 0.71356600 \\ \mathrm{H} & -2.40252700 & 2.09513800 & -1.04648800 \\ & & & \\ \mathrm{H} & & & \\ & & & \end{array}$

\section{2 a ketone}

Energy: -877.610687 au

Sum of electronic and thermal Energies: -877.347428 au Geometry:

$\begin{array}{rrrr}\mathrm{C} & 0.29765500 & 1.69892100 & 0.37440200 \\ \mathrm{H} & -0.04545900 & 2.68271600 & 0.67060500 \\ \mathrm{C} & -0.65934300 & 0.74376900 & 0.00286400 \\ \mathrm{C} & -0.21792200 & -0.52843200 & -0.38164700 \\ \mathrm{H} & -0.92458700 & -1.29344400 & -0.67709700 \\ \mathrm{C} & 1.13926800 & -0.84333200 & -0.39617000 \\ \mathrm{H} & 1.46341900 & -1.83136100 & -0.69694500 \\ \mathrm{C} & 2.06947800 & 0.12415500 & -0.02247700 \\ \mathrm{C} & 1.65384300 & 1.39971100 & 0.36460900 \\ \mathrm{H} & 2.37902900 & 2.14949000 & 0.65436500\end{array}$




\begin{tabular}{|c|c|c|c|}
\hline 0 & -2.40689400 & 2.29133200 & 0.34001600 \\
\hline $\mathrm{C}$ & -2.10957100 & 1.14955100 & 0.03817300 \\
\hline $\mathrm{C}$ & -4.47921900 & 0.77031200 & -0.78832200 \\
\hline $\mathrm{H}$ & -4.26348300 & 1.31818500 & -1.71089300 \\
\hline $\mathrm{H}$ & -4.81853200 & 1.51449300 & -0.06178700 \\
\hline $\mathrm{C}$ & -5.57988200 & -0.27154000 & -1.03050400 \\
\hline $\mathrm{H}$ & -6.49159500 & 0.22812900 & -1.37310900 \\
\hline $\mathrm{H}$ & -5.27455700 & -0.94676500 & -1.84107000 \\
\hline $\mathrm{C}$ & -4.59223600 & -1.74418200 & 0.78022200 \\
\hline $\mathrm{H}$ & -4.23261300 & -2.49793000 & 0.06737200 \\
\hline $\mathrm{H}$ & -4.80521300 & -2.27626800 & 1.71311800 \\
\hline $\mathrm{C}$ & -3.48641400 & -0.70693600 & 1.02150400 \\
\hline $\mathrm{H}$ & -3.79896400 & -0.01279300 & 1.81073900 \\
\hline $\mathrm{H}$ & -2.57699500 & -1.19975000 & 1.37861200 \\
\hline I & 4.12344700 & -0.34259100 & -0.04245300 \\
\hline $\mathrm{C}$ & -5.86980800 & -1.09545900 & 0.23142600 \\
\hline $\mathrm{H}$ & -6.62161900 & -1.86279700 & 0.02040200 \\
\hline $\mathrm{H}$ & -6.29925900 & -0.43970100 & 0.99957800 \\
\hline $\mathrm{C}$ & -3.19188300 & 0.11541400 & -0.26057200 \\
\hline $\mathrm{H}$ & -2.82092400 & -0.57725100 & -1.02507100 \\
\hline \multicolumn{4}{|c|}{ 2a hydrocarbon } \\
\hline \multicolumn{4}{|c|}{$\begin{array}{l}\text { Energy: }-803.574265 \text { au } \\
\text { Sum of electronic and thermal Energies: }-803.293187 \text { au } \\
\text { Geometry: }\end{array}$} \\
\hline $\mathrm{C}$ & -0.06448100 & 0.44253200 & 1.46475600 \\
\hline $\mathrm{H}$ & -0.54643900 & 0.29543000 & 2.42594000 \\
\hline $\mathrm{C}$ & -0.78683800 & 1.01029400 & 0.41112600 \\
\hline $\mathrm{C}$ & -0.12549200 & 1.19228100 & -0.80961900 \\
\hline $\mathrm{H}$ & -0.65437800 & 1.64106300 & -1.64484300 \\
\hline $\mathrm{C}$ & 1.20516200 & 0.81781000 & -0.98096900 \\
\hline $\mathrm{H}$ & 1.69451100 & 0.97308900 & -1.93431200 \\
\hline $\mathrm{C}$ & 1.89800800 & 0.24975000 & 0.08686600 \\
\hline $\mathrm{C}$ & 1.26902300 & 0.06109900 & 1.31414700 \\
\hline $\mathrm{H}$ & 1.80575500 & -0.37489700 & 2.14735100 \\
\hline $\mathrm{C}$ & -2.23734900 & 1.40949600 & 0.56941400 \\
\hline $\mathrm{C}$ & -4.66696000 & 1.05639300 & -0.07772600 \\
\hline $\mathrm{H}$ & -4.67149900 & 2.04285400 & -0.55524800 \\
\hline $\mathrm{H}$ & -4.95379900 & 1.21790400 & 0.97075100 \\
\hline $\mathrm{C}$ & -5.70031100 & 0.14077900 & -0.74962400 \\
\hline $\mathrm{H}$ & -6.70059800 & 0.57769700 & -0.66241200 \\
\hline $\mathrm{H}$ & -5.48165700 & 0.07800100 & -1.82365800 \\
\hline $\mathrm{C}$ & -4.26938300 & -1.87234800 & -0.18655100 \\
\hline $\mathrm{H}$ & -3.97571800 & -2.03820500 & -1.23130300 \\
\hline $\mathrm{H}$ & -4.26193700 & -2.85517100 & 0.29604500 \\
\hline $\mathrm{C}$ & -3.24089200 & -0.95116700 & 0.48590500 \\
\hline $\mathrm{H}$ & -3.47041000 & -0.87653300 & 1.55848700 \\
\hline $\mathrm{H}$ & -2.24086300 & -1.38873700 & 0.41168800 \\
\hline I & 3.91629800 & -0.31905300 & -0.15764700 \\
\hline $\mathrm{C}$ & -5.68060300 & -1.27076000 & -0.14757500 \\
\hline $\mathrm{H}$ & -6.38533700 & -1.91923700 & -0.67839800 \\
\hline $\mathrm{H}$ & -6.02434200 & -1.22366800 & 0.89418400 \\
\hline $\mathrm{H}$ & -2.48309100 & 1.47331300 & 1.63537100 \\
\hline $\mathrm{H}$ & -2.37537300 & 2.41679200 & 0.15959600 \\
\hline $\mathrm{C}$ & -3.24731700 & 0.46298700 & -0.11981200 \\
\hline $\mathrm{H}$ & -2.95402000 & 0.37522000 & -1.17599200 \\
\hline \multicolumn{4}{|c|}{$\begin{array}{l}\text { Energy: }-3170.008628 \text { au } \\
\text { Sum of electronic and thermal Energies: }-3169.756444 \text { au } \\
\text { Geometry: }\end{array}$} \\
\hline
\end{tabular}




\begin{tabular}{|c|c|c|c|}
\hline $\mathrm{Br}$ & -4.34801400 & -0.47666500 & -0.01762000 \\
\hline $\mathrm{N}$ & 2.62185200 & 0.40399400 & 0.12504500 \\
\hline 0 & 1.80499200 & 2.45113300 & -0.45873000 \\
\hline $\mathrm{C}$ & 1.60179500 & 1.28745300 & -0.13163500 \\
\hline $\mathrm{C}$ & 2.49566700 & -0.80659400 & 0.94586600 \\
\hline $\mathrm{H}$ & 1.44706000 & -1.07608900 & 1.04317600 \\
\hline $\mathrm{H}$ & 2.86746000 & -0.58048300 & 1.95704300 \\
\hline $\mathrm{C}$ & 4.00508600 & 0.86378200 & -0.05080000 \\
\hline $\mathrm{H}$ & 4.41369600 & 1.15946400 & 0.92683100 \\
\hline $\mathrm{H}$ & 3.98342200 & 1.75318800 & -0.67663500 \\
\hline $\mathrm{C}$ & 3.31397700 & -1.95859700 & 0.35471100 \\
\hline $\mathrm{H}$ & 3.24762700 & -2.82374700 & 1.02194900 \\
\hline $\mathrm{H}$ & 2.87472600 & -2.25601800 & -0.60461300 \\
\hline $\mathrm{C}$ & 4.86408300 & -0.24591700 & -0.66508900 \\
\hline $\mathrm{H}$ & 4.52413900 & -0.42913500 & -1.69080300 \\
\hline $\mathrm{H}$ & 5.90048000 & 0.09994400 & -0.72829500 \\
\hline $\mathrm{C}$ & 0.18256200 & 0.78336700 & -0.05827000 \\
\hline $\mathrm{C}$ & -0.76708400 & 1.57081400 & 0.59894100 \\
\hline $\mathrm{H}$ & -0.45093600 & 2.48763700 & 1.08182100 \\
\hline $\mathrm{C}$ & -2.10753600 & 1.19780000 & 0.62681700 \\
\hline $\mathrm{H}$ & -2.83610200 & 1.80477600 & 1.14848400 \\
\hline $\mathrm{C}$ & -2.50147900 & 0.03991900 & -0.03832400 \\
\hline $\mathrm{C}$ & -1.58138000 & -0.74453200 & -0.72648200 \\
\hline $\mathrm{H}$ & -1.90472200 & -1.63019200 & -1.25798300 \\
\hline $\mathrm{C}$ & -0.23925600 & -0.37019300 & -0.72574400 \\
\hline $\mathrm{H}$ & 0.47750000 & -0.97406800 & -1.27055300 \\
\hline $\mathrm{C}$ & 4.77685700 & -1.54325400 & 0.14931300 \\
\hline $\mathrm{H}$ & 5.24790400 & -1.38915300 & 1.12874500 \\
\hline $\mathrm{H}$ & 5.33559000 & -2.34416500 & -0.34376700 \\
\hline \multicolumn{4}{|c|}{$2 \mathrm{~b}$ amine } \\
\hline \multicolumn{4}{|c|}{ Energy: -3095.947556 au } \\
\hline \multicolumn{4}{|c|}{ Geometry: } \\
\hline $\mathrm{Br}$ & -4.27859900 & -0.48445100 & -0.02309500 \\
\hline $\mathrm{N}$ & 2.62624800 & 0.38865200 & -0.23414800 \\
\hline $\mathrm{C}$ & 1.63893100 & 1.46145000 & -0.14691700 \\
\hline $\mathrm{C}$ & 2.71499200 & -0.38484200 & 1.00859900 \\
\hline $\mathrm{H}$ & 1.71217400 & -0.72503500 & 1.27687300 \\
\hline $\mathrm{H}$ & 3.07200000 & 0.25769000 & 1.83912300 \\
\hline $\mathrm{C}$ & 3.93960800 & 0.88750900 & -0.64968400 \\
\hline $\mathrm{H}$ & 4.35560100 & 1.58548500 & 0.10499500 \\
\hline $\mathrm{H}$ & 3.81233200 & 1.45721400 & -1.57501100 \\
\hline $\mathrm{C}$ & 3.65386500 & -1.58264500 & 0.85208700 \\
\hline $\mathrm{H}$ & 3.72005100 & -2.11651500 & 1.80546500 \\
\hline $\mathrm{H}$ & 3.22240900 & -2.27606500 & 0.12170200 \\
\hline $\mathrm{C}$ & 4.92489600 & -0.26199500 & -0.87136400 \\
\hline $\mathrm{H}$ & 4.57316100 & -0.86978400 & -1.71243500 \\
\hline $\mathrm{H}$ & 5.90079200 & 0.14713100 & -1.15203000 \\
\hline $\mathrm{C}$ & 0.20712900 & 0.96384200 & -0.09007500 \\
\hline $\mathrm{C}$ & -0.70712300 & 1.50999000 & 0.81235400 \\
\hline $\mathrm{H}$ & -0.38135100 & 2.27358800 & 1.51153700 \\
\hline $\mathrm{C}$ & -2.03823200 & 1.09212300 & 0.83908400 \\
\hline $\mathrm{H}$ & -2.73620000 & 1.52279100 & 1.54549400 \\
\hline $\mathrm{C}$ & -2.45157800 & 0.10795100 & -0.05017700 \\
\hline $\mathrm{C}$ & -1.56152300 & -0.45980700 & -0.95831600 \\
\hline $\mathrm{H}$ & -1.89610000 & -1.22716700 & -1.64476700 \\
\hline $\mathrm{C}$ & -0.23867100 & -0.02650200 & -0.97190700 \\
\hline $\mathrm{H}$ & 0.46599800 & -0.46892900 & -1.66660300 \\
\hline $\mathrm{C}$ & 5.04326400 & -1.13567800 & 0.38309300 \\
\hline $\mathrm{H}$ & 5.52761100 & -0.55925800 & 1.18191800 \\
\hline
\end{tabular}




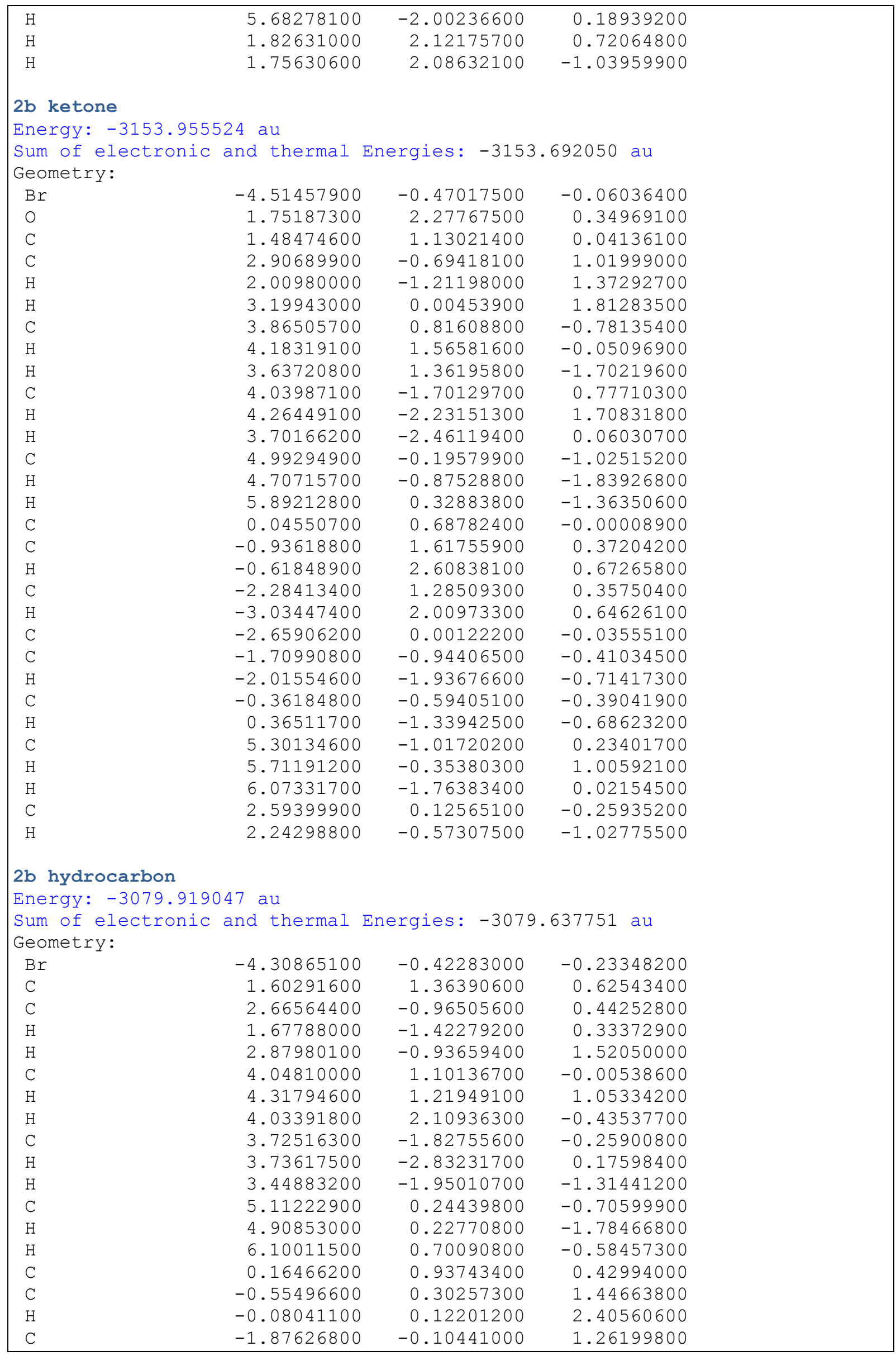




\begin{tabular}{|c|c|c|c|}
\hline $\mathrm{H}$ & -2.41765700 & -0.59308200 & 2.06195800 \\
\hline $\mathrm{C}$ & -2.48890200 & 0.12908900 & 0.03677100 \\
\hline $\mathrm{C}$ & -1.80480500 & 0.76275400 & -0.99620300 \\
\hline $\mathrm{H}$ & -2.29326100 & 0.94771700 & -1.94440000 \\
\hline $\mathrm{C}$ & -0.48644000 & 1.16102400 & -0.78951700 \\
\hline $\mathrm{H}$ & 0.04081000 & 1.66183000 & -1.59546000 \\
\hline $\mathrm{C}$ & 5.12031200 & -1.19434500 & -0.17201000 \\
\hline $\mathrm{H}$ & 5.44955800 & -1.18874800 & 0.87546300 \\
\hline $\mathrm{H}$ & 5.84767300 & -1.79932800 & -0.72325700 \\
\hline $\mathrm{H}$ & 1.83453000 & 1.38276200 & 1.69627300 \\
\hline $\mathrm{H}$ & 1.72066400 & 2.39272700 & 0.26587500 \\
\hline $\mathrm{C}$ & 2.64428500 & 0.47634600 & -0.09469400 \\
\hline $\mathrm{H}$ & 2.36609500 & 0.43235300 & -1.15768700 \\
\hline \multicolumn{4}{|l|}{$2 c$} \\
\hline \multicolumn{4}{|c|}{ Energy: -1056.088984 au } \\
\hline $\mathrm{N}$ & 1.91626000 & 0.42644000 & 0.12512400 \\
\hline 0 & 0.99334700 & 2.42999900 & -0.45149000 \\
\hline $\mathrm{C}$ & 0.85098800 & 1.25591000 & -0.12934900 \\
\hline $\mathrm{C}$ & 1.85400500 & -0.78971400 & 0.94498400 \\
\hline $\mathrm{H}$ & 0.82109500 & -1.11438400 & 1.04161100 \\
\hline $\mathrm{H}$ & 2.21281600 & -0.54498300 & 1.95655000 \\
\hline $\mathrm{C}$ & 3.27325100 & 0.95925700 & -0.04846700 \\
\hline $\mathrm{H}$ & 3.66468800 & 1.27494000 & 0.92996100 \\
\hline $\mathrm{H}$ & 3.20516500 & 1.84711500 & -0.67316800 \\
\hline $\mathrm{C}$ & 2.73249900 & -1.89623600 & 0.35344100 \\
\hline $\mathrm{H}$ & 2.71172900 & -2.76430100 & 1.01988600 \\
\hline $\mathrm{H}$ & 2.31018800 & -2.21566500 & -0.60642400 \\
\hline $\mathrm{C}$ & 4.19054300 & -0.10251900 & -0.66324400 \\
\hline $\mathrm{H}$ & 3.86179800 & -0.30213000 & -1.68955300 \\
\hline $\mathrm{H}$ & 5.20722200 & 0.29786300 & -0.72492300 \\
\hline $\mathrm{C}$ & -0.53942900 & 0.67723900 & -0.05981300 \\
\hline $\mathrm{C}$ & -1.53146300 & 1.41368700 & 0.59427700 \\
\hline $\mathrm{H}$ & -1.26532300 & 2.34621900 & 1.07703200 \\
\hline $\mathrm{C}$ & -2.84962700 & 0.96999400 & 0.61890600 \\
\hline $\mathrm{H}$ & -3.61438200 & 1.53466200 & 1.13662400 \\
\hline $\mathrm{C}$ & -3.18010600 & -0.20756400 & -0.04594000 \\
\hline $\mathrm{C}$ & -2.21774900 & -0.94231200 & -0.73090200 \\
\hline $\mathrm{H}$ & -2.49709700 & -1.84377100 & -1.26111000 \\
\hline $\mathrm{C}$ & -0.89787000 & -0.49766600 & -0.72738400 \\
\hline $\mathrm{H}$ & -0.14861100 & -1.06278400 & -1.26985700 \\
\hline $\mathrm{Cl}$ & -4.84555800 & -0.77034100 & -0.03064200 \\
\hline $\mathrm{C}$ & 4.17150500 & -1.40380500 & 0.14939000 \\
\hline $\mathrm{H}$ & 4.77210700 & -2.17339100 & -0.34436600 \\
\hline $\mathrm{H}$ & 4.63320900 & -1.22630400 & 1.12931500 \\
\hline \multicolumn{4}{|c|}{ 2c amine } \\
\hline Energy: -982.027773 au & \multicolumn{3}{|c|}{$\begin{array}{l}\text { Sum of electronic and thermal Energies: }-981.757960 \text { au } \\
\text { Geometry: }\end{array}$} \\
\hline $\mathrm{N}$ & 1.89904500 & 0.39948400 & -0.23057800 \\
\hline $\mathrm{C}$ & 0.85995000 & 1.42178200 & -0.13621000 \\
\hline $\mathrm{C}$ & 2.02236900 & -0.38091200 & 1.00481300 \\
\hline $\mathrm{H}$ & 1.03662800 & -0.77204600 & 1.26683700 \\
\hline $\mathrm{H}$ & 2.34571400 & 0.26999700 & 1.84259800 \\
\hline $\mathrm{C}$ & 3.18741500 & 0.96623800 & -0.63689400 \\
\hline $\mathrm{H}$ & 3.56664700 & 1.67611800 & 0.12603700 \\
\hline $\mathrm{H}$ & 3.03495200 & 1.53836000 & -1.55690400 \\
\hline $\mathrm{C}$ & 3.01876100 & -1.52994700 & 0.83920900 \\
\hline
\end{tabular}




\begin{tabular}{|c|c|c|c|}
\hline $\mathrm{H}$ & 3.10853600 & -2.06963100 & 1.78738200 \\
\hline $\mathrm{H}$ & 2.62333300 & -2.23606200 & 0.10063900 \\
\hline $\mathrm{C}$ & 4.22854100 & -0.13129200 & -0.86693700 \\
\hline $\mathrm{H}$ & 3.90943000 & -0.74680400 & -1.71537000 \\
\hline $\mathrm{H}$ & 5.18404500 & 0.32799700 & -1.14008400 \\
\hline $\mathrm{C}$ & -0.54575200 & 0.85403200 & -0.08700400 \\
\hline $\mathrm{C}$ & -1.48635500 & 1.34326200 & 0.82109000 \\
\hline $\mathrm{H}$ & -1.19867600 & 2.11313900 & 1.52989800 \\
\hline $\mathrm{C}$ & -2.79470400 & 0.85996500 & 0.84092300 \\
\hline $\mathrm{H}$ & -3.51687100 & 1.24394800 & 1.55019600 \\
\hline $\mathrm{C}$ & -3.15915400 & -0.13207000 & -0.06061300 \\
\hline $\mathrm{C}$ & -2.24156200 & -0.64358200 & -0.97451300 \\
\hline $\mathrm{H}$ & -2.54224400 & -1.41795500 & -1.66902000 \\
\hline $\mathrm{C}$ & -0.94209400 & -0.14593800 & -0.98172800 \\
\hline $\mathrm{H}$ & -0.21616300 & -0.54435000 & -1.68103800 \\
\hline $\mathrm{Cl}$ & -4.80577100 & -0.75763000 & -0.04421800 \\
\hline $\mathrm{C}$ & 4.38598700 & -1.01117300 & 0.37891100 \\
\hline $\mathrm{H}$ & 5.06743700 & -1.84367400 & 0.17834400 \\
\hline $\mathrm{H}$ & 4.83963800 & -0.42015000 & 1.18506100 \\
\hline $\mathrm{H}$ & 1.01318800 & 2.08244400 & 0.73778100 \\
\hline $\mathrm{H}$ & 0.94878000 & 2.05979100 & -1.02287700 \\
\hline \multicolumn{4}{|c|}{ 2c ketone } \\
\hline \multicolumn{4}{|c|}{ Energy: -1040.035962 au } \\
\hline \multicolumn{4}{|c|}{ Geometry: } \\
\hline 0 & 0.93706500 & 2.23903300 & 0.37172600 \\
\hline $\mathrm{C}$ & 0.72355900 & 1.08394700 & 0.04991200 \\
\hline $\mathrm{C}$ & 2.22982200 & -0.67751200 & 1.01867700 \\
\hline $\mathrm{H}$ & 1.35815300 & -1.24010500 & 1.36684200 \\
\hline $\mathrm{H}$ & 2.48739300 & 0.02923800 & 1.81650000 \\
\hline $\mathrm{C}$ & 3.11644100 & 0.88841100 & -0.77188700 \\
\hline $\mathrm{H}$ & 3.39734300 & 1.64807600 & -0.03654600 \\
\hline $\mathrm{H}$ & 2.86350300 & 1.42828600 & -1.68973000 \\
\hline $\mathrm{C}$ & 3.41025800 & -1.62734400 & 0.77098000 \\
\hline $\mathrm{H}$ & 3.65910400 & -2.15217300 & 1.69909300 \\
\hline $\mathrm{H}$ & 3.10988700 & -2.39797900 & 0.04881500 \\
\hline $\mathrm{C}$ & 4.29198700 & -0.06641100 & -1.02040500 \\
\hline $\mathrm{H}$ & 4.04019800 & -0.75379600 & -1.83910800 \\
\hline $\mathrm{H}$ & 5.16528700 & 0.50299400 & -1.35414800 \\
\hline $\mathrm{C}$ & -0.69334300 & 0.57730800 & -0.00437000 \\
\hline $\mathrm{C}$ & -1.71924100 & 1.46079300 & 0.36204200 \\
\hline $\mathrm{H}$ & -1.44873800 & 2.46383400 & 0.66795400 \\
\hline $\mathrm{C}$ & -3.04992600 & 1.06788900 & 0.33476000 \\
\hline $\mathrm{H}$ & -3.83816900 & 1.75374800 & 0.61785400 \\
\hline $\mathrm{C}$ & -3.36355400 & -0.23029200 & -0.06448900 \\
\hline $\mathrm{C}$ & -2.36950400 & -1.13053900 & -0.43338700 \\
\hline $\mathrm{H}$ & -2.63243900 & -2.13429200 & -0.74139400 \\
\hline $\mathrm{C}$ & -1.03935300 & -0.72060200 & -0.40142200 \\
\hline $\mathrm{H}$ & -0.27688500 & -1.43131000 & -0.69284300 \\
\hline $\mathrm{Cl}$ & -5.04300800 & -0.73807000 & -0.10294100 \\
\hline $\mathrm{C}$ & 4.63798000 & -0.87979100 & 0.23417000 \\
\hline $\mathrm{H}$ & 5.44538600 & -1.58698000 & 0.01844400 \\
\hline $\mathrm{H}$ & 5.01494000 & -0.20215800 & 1.01083900 \\
\hline $\mathrm{C}$ & 1.87933700 & 0.13457700 & -0.25578800 \\
\hline $\mathrm{H}$ & 1.56285500 & -0.57505200 & -1.02915500 \\
\hline \multicolumn{3}{|c|}{$\begin{array}{l}\text { Sum of electronic and thermal Energies: }-965.717636 \mathrm{au} \\
\text { Geometry: }\end{array}$} & 17636 au \\
\hline
\end{tabular}




\begin{tabular}{|c|c|c|c|}
\hline $\mathrm{C}$ & 0.81973100 & 1.26730400 & 0.71624100 \\
\hline $\mathrm{C}$ & 1.98259100 & -0.99246700 & 0.35858900 \\
\hline $\mathrm{H}$ & 1.01734200 & -1.47730700 & 0.18463600 \\
\hline $\mathrm{H}$ & 2.17118100 & -1.05084800 & 1.44013700 \\
\hline $\mathrm{C}$ & 3.28744400 & 1.15834100 & 0.12936500 \\
\hline $\mathrm{H}$ & 3.52814100 & 1.19239600 & 1.20108400 \\
\hline $\mathrm{H}$ & 3.24104600 & 2.19921600 & -0.21061500 \\
\hline $\mathrm{C}$ & 3.09255300 & -1.74704200 & -0.38786800 \\
\hline $\mathrm{H}$ & 3.13564600 & -2.78516400 & -0.04240300 \\
\hline $\mathrm{H}$ & 2.84533600 & -1.78639200 & -1.45679400 \\
\hline $\mathrm{C}$ & 4.40182900 & 0.40955800 & -0.61563800 \\
\hline $\mathrm{H}$ & 4.22332600 & 0.48037900 & -1.69657200 \\
\hline $\mathrm{H}$ & 5.36689300 & 0.89215700 & -0.42900800 \\
\hline $\mathrm{C}$ & -0.59483500 & 0.80262500 & 0.44797600 \\
\hline $\mathrm{C}$ & -1.30885700 & 0.05149300 & 1.38651900 \\
\hline $\mathrm{H}$ & -0.84766600 & -0.19432000 & 2.33738100 \\
\hline $\mathrm{C}$ & -2.60708300 & -0.38974600 & 1.13347600 \\
\hline $\mathrm{H}$ & -3.14837500 & -0.96929000 & 1.87059100 \\
\hline $\mathrm{C}$ & -3.20289200 & -0.07337300 & -0.08120800 \\
\hline $\mathrm{C}$ & -2.52392500 & 0.67660700 & -1.03647100 \\
\hline $\mathrm{H}$ & -3.00347700 & 0.92307500 & -1.97537300 \\
\hline $\mathrm{C}$ & -1.22869100 & 1.10749800 & -0.76302900 \\
\hline $\mathrm{H}$ & -0.70578400 & 1.69876100 & -1.50811500 \\
\hline $\mathrm{Cl}$ & -4.84311200 & -0.62253200 & -0.41463100 \\
\hline $\mathrm{C}$ & 4.45784700 & -1.06941600 & -0.20960900 \\
\hline $\mathrm{H}$ & 5.22191600 & -1.59401500 & -0.79265300 \\
\hline $\mathrm{H}$ & 4.76310500 & -1.14356100 & 0.84251300 \\
\hline $\mathrm{H}$ & 1.02680100 & 1.20137900 & 1.79026900 \\
\hline $\mathrm{H}$ & 0.90228300 & 2.32763100 & 0.45065200 \\
\hline $\mathrm{C}$ & 1.91326500 & 0.48879100 & -0.05104900 \\
\hline $\mathrm{H}$ & 1.66133400 & 0.52791000 & -1.12078100 \\
\hline \multicolumn{4}{|l|}{$2 d$} \\
\hline \multicolumn{4}{|c|}{$\begin{array}{l}\text { Sum of electronic and thermal Energies: }-695.481428 \text { au } \\
\text { Geometry: }\end{array}$} \\
\hline $\mathrm{C}$ & 0.96180900 & 0.55117600 & -0.05713900 \\
\hline $\mathrm{C}$ & 1.24157500 & -0.65049000 & -0.71620600 \\
\hline $\mathrm{H}$ & 0.45368200 & -1.17228200 & -1.24684100 \\
\hline $\mathrm{C}$ & 2.53205100 & -1.17505100 & -0.72647800 \\
\hline $\mathrm{H}$ & 2.76853100 & -2.09541600 & -1.24561600 \\
\hline $\mathrm{C}$ & 3.52986500 & -0.48461600 & -0.05678900 \\
\hline $\mathrm{C}$ & 3.29177300 & 0.71314400 & 0.60037200 \\
\hline $\mathrm{H}$ & 4.10503600 & 1.22410400 & 1.10038400 \\
\hline $\mathrm{C}$ & 2.00207200 & 1.23439700 & 0.58162300 \\
\hline $\mathrm{H}$ & 1.79404400 & 2.18587200 & 1.05546600 \\
\hline 0 & -0.46202800 & 2.39844000 & -0.43026100 \\
\hline $\mathrm{C}$ & -0.38986800 & 1.21418900 & -0.12188700 \\
\hline $\mathrm{N}$ & -1.50545200 & 0.44776900 & 0.12029300 \\
\hline $\mathrm{C}$ & -2.82582300 & 1.06496500 & -0.05577400 \\
\hline $\mathrm{H}$ & -2.70154100 & 1.94474200 & -0.68324900 \\
\hline $\mathrm{H}$ & -3.19752200 & 1.40861500 & 0.92104100 \\
\hline $\mathrm{C}$ & -3.80815000 & 0.06154800 & -0.66800800 \\
\hline $\mathrm{H}$ & -4.79723400 & 0.52559500 & -0.73255100 \\
\hline $\mathrm{H}$ & -3.49154000 & -0.16229500 & -1.69313800 \\
\hline $\mathrm{C}$ & -2.46851600 & -1.81745700 & 0.35685600 \\
\hline $\mathrm{H}$ & -2.06639000 & -2.16694700 & -0.60122700 \\
\hline $\mathrm{H}$ & -2.50371900 & -2.68244100 & 1.02674300 \\
\hline $\mathrm{C}$ & -1.52234400 & -0.76651600 & 0.94531200 \\
\hline $\mathrm{H}$ & -1.86689100 & -0.49538300 & 1.95524500 \\
\hline $\mathrm{H}$ & -0.51240600 & -1.15576400 & 1.04558600 \\
\hline
\end{tabular}




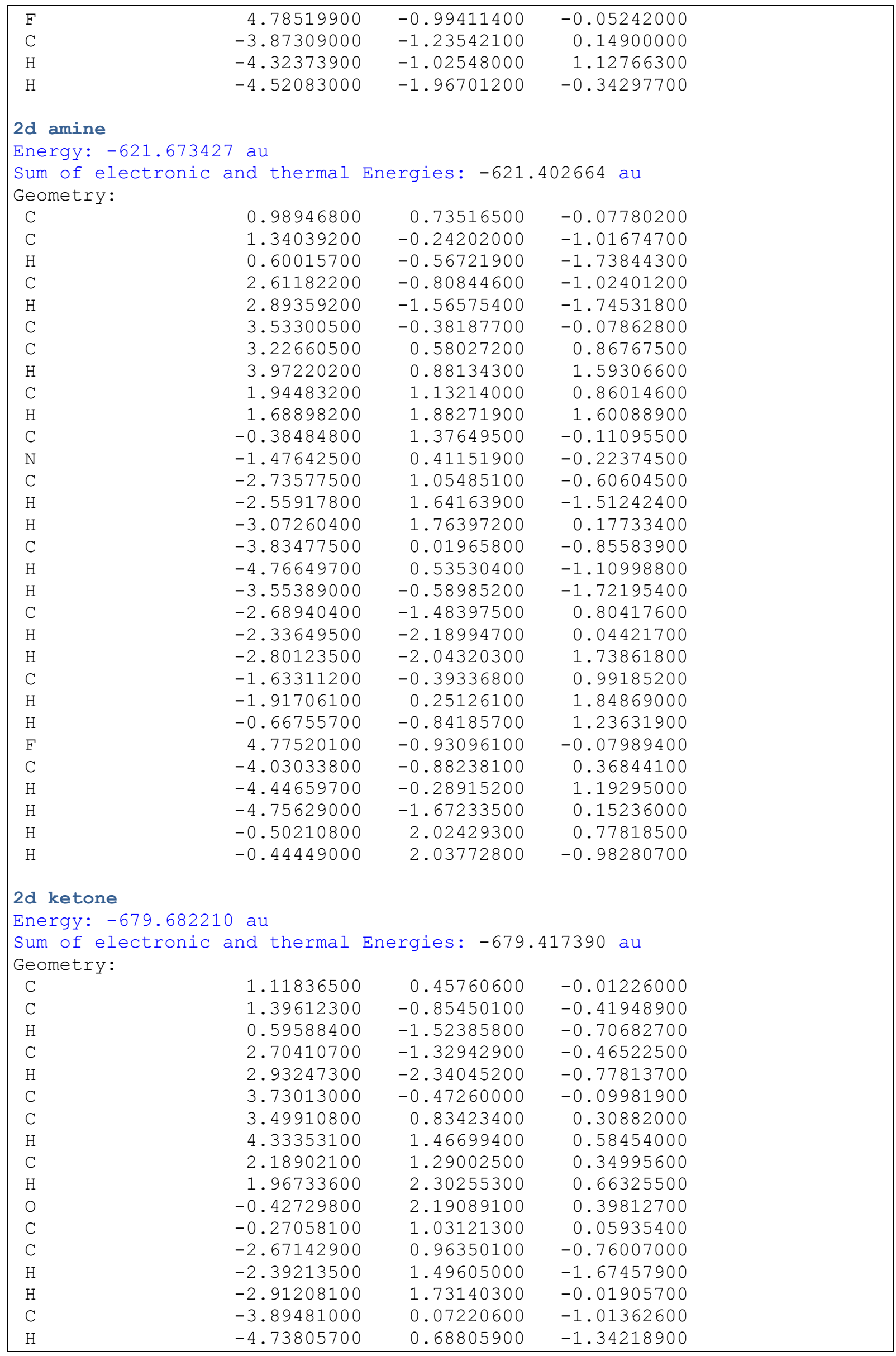




\begin{tabular}{|c|c|c|c|}
\hline $\mathrm{H}$ & -3.67957800 & -0.62159100 & -1.83735100 \\
\hline $\mathrm{C}$ & -3.09240800 & -1.54423400 & 0.76573300 \\
\hline $\mathrm{H}$ & -2.83276100 & -2.32420000 & 0.03779600 \\
\hline $\mathrm{H}$ & -3.36697700 & -2.06216500 & 1.69049300 \\
\hline $\mathrm{C}$ & -1.86450300 & -0.65801100 & 1.01825900 \\
\hline $\mathrm{H}$ & -2.08442700 & 0.05549200 & 1.82129900 \\
\hline $\mathrm{H}$ & -1.02250800 & -1.26710500 & 1.36130200 \\
\hline $\mathrm{F}$ & 5.00312000 & -0.92600200 & -0.14323100 \\
\hline $\mathrm{C}$ & -4.28076700 & -0.73101400 & 0.23585600 \\
\hline $\mathrm{H}$ & -4.62146600 & -0.04025300 & 1.01769800 \\
\hline $\mathrm{H}$ & -5.12369600 & -1.39431500 & 0.01655000 \\
\hline $\mathrm{C}$ & -1.47393200 & 0.14368100 & -0.25096400 \\
\hline $\mathrm{H}$ & -1.19578200 & -0.57578600 & -1.02986700 \\
\hline \multicolumn{4}{|c|}{ 2d hydrocarbon } \\
\hline \multicolumn{4}{|c|}{ Energy: -605.644804 au } \\
\hline \multicolumn{4}{|c|}{ Geometry: } \\
\hline $\mathrm{C}$ & 1.04381300 & 0.65257900 & 0.45689900 \\
\hline $\mathrm{C}$ & 1.65825300 & 1.06908300 & -0.73127300 \\
\hline $\mathrm{H}$ & 1.14086900 & 1.76201300 & -1.38703200 \\
\hline $\mathrm{C}$ & 2.92667200 & 0.62074400 & -1.09168500 \\
\hline $\mathrm{H}$ & 3.40604300 & 0.94436300 & -2.00737800 \\
\hline $\mathrm{C}$ & 3.58312900 & -0.25631200 & -0.24254600 \\
\hline $\mathrm{C}$ & 3.01892000 & -0.69126000 & 0.94420900 \\
\hline $\mathrm{H}$ & 3.56627500 & -1.37240500 & 1.58405500 \\
\hline $\mathrm{C}$ & 1.74730200 & -0.22931300 & 1.28384400 \\
\hline $\mathrm{H}$ & 1.29833800 & -0.56192700 & 2.21375200 \\
\hline $\mathrm{C}$ & -0.34273300 & 1.13992400 & 0.81887400 \\
\hline $\mathrm{C}$ & -2.82867700 & 1.19399700 & 0.30424600 \\
\hline $\mathrm{H}$ & -2.74811100 & 2.26423400 & 0.08218000 \\
\hline $\mathrm{H}$ & -3.03774700 & 1.11542600 & 1.38027100 \\
\hline $\mathrm{C}$ & -3.99406800 & 0.57851600 & -0.48358400 \\
\hline $\mathrm{H}$ & -4.93250900 & 1.07333400 & -0.21226000 \\
\hline $\mathrm{H}$ & -3.84323600 & 0.76529300 & -1.55489200 \\
\hline $\mathrm{C}$ & -2.77040500 & -1.63882900 & -0.54321700 \\
\hline $\mathrm{H}$ & -2.55492900 & -1.56556300 & -1.61722700 \\
\hline $\mathrm{H}$ & -2.84753600 & -2.70717100 & -0.31589000 \\
\hline $\mathrm{C}$ & -1.60954700 & -1.01754900 & 0.24730700 \\
\hline $\mathrm{H}$ & -1.77042000 & -1.19163900 & 1.32090100 \\
\hline $\mathrm{H}$ & -0.67054600 & -1.51602500 & -0.01110800 \\
\hline $\mathrm{F}$ & 4.82165600 & -0.69762600 & -0.58399100 \\
\hline $\mathrm{C}$ & -4.10057200 & -0.93392200 & -0.24547600 \\
\hline $\mathrm{H}$ & -4.37903100 & -1.11569400 & 0.80098500 \\
\hline $\mathrm{H}$ & -4.90204200 & -1.35885100 & -0.85873000 \\
\hline $\mathrm{H}$ & -0.52353500 & 0.96204400 & 1.88499300 \\
\hline $\mathrm{H}$ & -0.38749700 & 2.22596300 & 0.67658200 \\
\hline $\mathrm{C}$ & -1.48930200 & 0.49695700 & 0.00534200 \\
\hline $\mathrm{H}$ & -1.26599800 & 0.64757200 & -1.06092800 \\
\hline \multicolumn{4}{|l|}{$2 e$} \\
\hline \multicolumn{4}{|c|}{$\begin{array}{l}\text { Sum of electronic and thermal Energies: } \mathbf{- 5 9 6 . 2 0 5 3 0 3 ~ a u ~} \\
\text { Geometry: }\end{array}$} \\
\hline $\mathrm{C}$ & 1.37076300 & 0.36783500 & -0.07294900 \\
\hline $\mathrm{C}$ & 1.55865600 & -0.83237600 & -0.76651300 \\
\hline $\mathrm{H}$ & 0.73576500 & -1.26810300 & -1.32220500 \\
\hline $\mathrm{C}$ & 2.80494500 & -1.45590100 & -0.77048400 \\
\hline $\mathrm{H}$ & 2.94478700 & -2.37744500 & -1.32452000 \\
\hline $\mathrm{C}$ & 3.87001100 & -0.89343000 & -0.06885700 \\
\hline $\mathrm{C}$ & 3.68979000 & 0.30617800 & 0.61959000 \\
\hline
\end{tabular}




\begin{tabular}{|c|c|c|c|}
\hline $\mathrm{H}$ & 4.51796700 & 0.75290900 & 1.15822400 \\
\hline $\mathrm{C}$ & 2.45114300 & 0.94139800 & 0.60479300 \\
\hline $\mathrm{H}$ & 2.31265100 & 1.89106100 & 1.10773700 \\
\hline 0 & 0.09341500 & 2.31976300 & -0.45521500 \\
\hline $\mathrm{C}$ & 0.07528300 & 1.13676000 & -0.13406500 \\
\hline $\mathrm{N}$ & -1.09465500 & 0.46442400 & 0.12934800 \\
\hline $\mathrm{C}$ & -2.36480800 & 1.18276000 & -0.02623500 \\
\hline $\mathrm{H}$ & -2.17819900 & 2.05913000 & -0.64300400 \\
\hline $\mathrm{H}$ & -2.70102800 & 1.53943400 & 0.95882500 \\
\hline $\mathrm{C}$ & -3.42690300 & 0.26650300 & -0.64217500 \\
\hline $\mathrm{H}$ & -4.37837600 & 0.80536200 & -0.69002500 \\
\hline $\mathrm{H}$ & -3.13782800 & 0.03385600 & -1.67346200 \\
\hline $\mathrm{C}$ & -2.22482700 & -1.72388400 & 0.34277100 \\
\hline $\mathrm{H}$ & -1.85934900 & -2.08835200 & -0.62428000 \\
\hline $\mathrm{H}$ & -2.31990800 & -2.59373000 & 1.00054100 \\
\hline $\mathrm{C}$ & -1.19479500 & -0.75746700 & 0.93579300 \\
\hline $\mathrm{H}$ & -1.50618200 & -0.47772600 & 1.95417600 \\
\hline $\mathrm{H}$ & -0.21622700 & -1.22393300 & 1.01569600 \\
\hline $\mathrm{H}$ & 4.83791800 & -1.38201500 & -0.06674700 \\
\hline $\mathrm{C}$ & -3.58272000 & -1.03336300 & 0.15796900 \\
\hline $\mathrm{H}$ & -4.28950900 & -1.70625600 & -0.33668900 \\
\hline $\mathrm{H}$ & -4.00644500 & -0.80334200 & 1.14420000 \\
\hline \multicolumn{4}{|c|}{$2 e$ amine } \\
\hline $\begin{array}{l}\text { Sum of electronic and thermal Energies: }-522.126331 \text { au } \\
\text { Geometry: }\end{array}$ & $\begin{array}{l}9 \text { au } \\
\text { and thermal }\end{array}$ & rgies: -522 & 26331 au \\
\hline $\mathrm{C}$ & -1.42930700 & -0.56431000 & -0.07073400 \\
\hline $\mathrm{C}$ & -1.70807300 & 0.39947700 & -1.04680100 \\
\hline $\mathrm{H}$ & -0.94165800 & 0.64791100 & -1.77224300 \\
\hline $\mathrm{C}$ & -2.94293800 & 1.04185100 & -1.07791000 \\
\hline $\mathrm{H}$ & -3.14370800 & 1.78689500 & -1.84028000 \\
\hline $\mathrm{C}$ & -3.92232000 & 0.72960300 & -0.13371700 \\
\hline $\mathrm{C}$ & -3.65454100 & -0.22684300 & 0.84237100 \\
\hline $\mathrm{H}$ & -4.40643600 & -0.47296300 & 1.58420700 \\
\hline $\mathrm{C}$ & -2.41422000 & -0.86569800 & 0.87271600 \\
\hline $\mathrm{H}$ & -2.20931200 & -1.60526500 & 1.64067700 \\
\hline $\mathrm{C}$ & -0.10031000 & -1.29622000 & -0.06691900 \\
\hline $\mathrm{N}$ & 1.05586800 & -0.41375000 & -0.21043100 \\
\hline $\mathrm{C}$ & 2.27140400 & -1.15803500 & -0.54624600 \\
\hline $\mathrm{H}$ & 2.06380200 & -1.77362800 & -1.42661700 \\
\hline $\mathrm{H}$ & 2.55183600 & -1.85100500 & 0.27331500 \\
\hline C & 3.44115700 & -0.21289300 & -0.82986000 \\
\hline $\mathrm{H}$ & 4.33801000 & -0.80192000 & -1.04772300 \\
\hline $\mathrm{H}$ & 3.21080100 & 0.37265600 & -1.72688100 \\
\hline $\mathrm{C}$ & 2.38503300 & 1.44140900 & 0.74394800 \\
\hline $\mathrm{H}$ & 2.08810500 & 2.13295400 & -0.05234600 \\
\hline $\mathrm{H}$ & 2.52595400 & 2.03517300 & 1.65288200 \\
\hline $\mathrm{C}$ & 1.25512700 & 0.43461800 & 0.96832200 \\
\hline $\mathrm{H}$ & 1.48637400 & -0.18658200 & 1.85790800 \\
\hline $\mathrm{H}$ & 0.31997800 & 0.95815600 & 1.17912200 \\
\hline $\mathrm{H}$ & -4.88376600 & 1.23039100 & -0.15844700 \\
\hline $\mathrm{C}$ & 3.68609800 & 0.73082800 & 0.35348400 \\
\hline $\mathrm{H}$ & 4.46628200 & 1.45884000 & 0.11010100 \\
\hline $\mathrm{H}$ & 4.05315600 & 0.15036600 & 1.20988900 \\
\hline $\mathrm{H}$ & -0.03085900 & -1.91352900 & 0.84866200 \\
\hline $\mathrm{H}$ & -0.08228500 & -1.99493300 & -0.91113700 \\
\hline \multicolumn{4}{|c|}{$2 e$ ketone } \\
\hline \multicolumn{4}{|c|}{ Energy: -580.413101 au } \\
\hline \multicolumn{4}{|c|}{ Sum of electronic and thermal Energies: -580.140875 au } \\
\hline
\end{tabular}




\begin{tabular}{|c|c|c|c|}
\hline \multicolumn{4}{|c|}{ Geometry: } \\
\hline $\mathrm{C}$ & 1.53393400 & 0.28907400 & -0.03392700 \\
\hline $\mathrm{C}$ & 1.71896200 & -1.02873800 & -0.47366300 \\
\hline $\mathrm{H}$ & 0.87234000 & -1.63347000 & -0.77260300 \\
\hline $\mathrm{C}$ & 2.99608400 & -1.58274100 & -0.53504200 \\
\hline $\mathrm{H}$ & 3.12482200 & -2.60325200 & -0.87758900 \\
\hline $\mathrm{C}$ & 4.10427100 & -0.82682400 & -0.15907700 \\
\hline $\mathrm{C}$ & 3.93212100 & 0.48714900 & 0.28028100 \\
\hline $\mathrm{H}$ & 4.79247300 & 1.07832400 & 0.57332100 \\
\hline $\mathrm{C}$ & 2.65867000 & 1.03871900 & 0.34211100 \\
\hline $\mathrm{H}$ & 2.50593400 & 2.05631700 & 0.67983600 \\
\hline 0 & 0.10607000 & 2.11611000 & 0.41057100 \\
\hline $\mathrm{C}$ & 0.18474700 & 0.95314700 & 0.05675200 \\
\hline $\mathrm{C}$ & -2.22862400 & 1.05425800 & -0.72491300 \\
\hline $\mathrm{H}$ & -1.93002600 & 1.59069500 & -1.63101300 \\
\hline $\mathrm{H}$ & -2.41119100 & 1.81758900 & 0.03717800 \\
\hline $\mathrm{C}$ & -3.50818000 & 0.24601700 & -0.97996800 \\
\hline $\mathrm{H}$ & -4.31661600 & 0.91980200 & -1.28147300 \\
\hline $\mathrm{H}$ & -3.34742600 & -0.44003900 & -1.82241600 \\
\hline $\mathrm{C}$ & -2.78212500 & -1.45828200 & 0.74955000 \\
\hline $\mathrm{H}$ & -2.58105000 & -2.23520200 & 0.00012700 \\
\hline $\mathrm{H}$ & -3.07528000 & -1.98021100 & 1.66640900 \\
\hline $\mathrm{C}$ & -1.49853900 & -0.65508800 & 1.00289700 \\
\hline $\mathrm{H}$ & -1.66316900 & 0.05157100 & 1.82513000 \\
\hline $\mathrm{H}$ & -0.69080000 & -1.32218100 & 1.31876500 \\
\hline $\mathrm{H}$ & 5.09785200 & -1.25836000 & -0.20829500 \\
\hline $\mathrm{C}$ & -3.92555400 & -0.56173000 & 0.25654600 \\
\hline $\mathrm{H}$ & -4.81074500 & -1.16679200 & 0.03496200 \\
\hline $\mathrm{H}$ & -4.21229700 & 0.12991800 & 1.05904600 \\
\hline $\mathrm{C}$ & -1.07714500 & 0.15054100 & -0.25379400 \\
\hline $\mathrm{H}$ & -0.85511300 & -0.56660600 & -1.05245800 \\
\hline \multicolumn{4}{|c|}{ 2e hydrocarbon } \\
\hline \multicolumn{4}{|c|}{ Energy: -506.376068 au } \\
\hline \multicolumn{4}{|c|}{$\begin{array}{l}\text { Sum of electronic and thermal Energies: }-506.086102 \text { au } \\
\text { Geometry: }\end{array}$} \\
\hline $\mathrm{C}$ & -1.48852500 & -0.47289200 & 0.41573300 \\
\hline $\mathrm{C}$ & -2.07047000 & -1.00808300 & -0.74096100 \\
\hline $\mathrm{H}$ & -1.55640700 & -1.79800300 & -1.28031300 \\
\hline $\mathrm{C}$ & -3.30079100 & -0.54968800 & -1.20579900 \\
\hline $\mathrm{H}$ & -3.73372900 & -0.98176900 & -2.10154400 \\
\hline $\mathrm{C}$ & -3.97871100 & 0.45707600 & -0.51844100 \\
\hline $\mathrm{C}$ & -3.41574400 & 0.99571000 & 0.63623900 \\
\hline $\mathrm{H}$ & -3.93620100 & 1.77521000 & 1.18207700 \\
\hline $\mathrm{C}$ & -2.18295000 & 0.53279900 & 1.09627100 \\
\hline $\mathrm{H}$ & -1.75504400 & 0.95677300 & 1.99923100 \\
\hline $\mathrm{C}$ & -0.14293500 & -0.96896100 & 0.89957700 \\
\hline $\mathrm{C}$ & 2.35572400 & -1.19674800 & 0.50573900 \\
\hline $\mathrm{H}$ & 2.23182900 & -2.28223200 & 0.41840500 \\
\hline $\mathrm{H}$ & 2.52743600 & -0.98898500 & 1.57116400 \\
\hline $\mathrm{C}$ & 3.57880500 & -0.73931200 & -0.30209300 \\
\hline $\mathrm{H}$ & 4.48133400 & -1.23494500 & 0.07087600 \\
\hline $\mathrm{H}$ & 3.45982500 & -1.05623400 & -1.34651900 \\
\hline $\mathrm{C}$ & 2.46698900 & 1.50235100 & -0.69723500 \\
\hline $\mathrm{H}$ & 2.28900000 & 1.29988000 & -1.76152100 \\
\hline $\mathrm{H}$ & 2.58734100 & 2.58687200 & -0.60505000 \\
\hline $\mathrm{C}$ & 1.24829200 & 1.03931400 & 0.11447100 \\
\hline $\mathrm{H}$ & 1.37661300 & 1.34346100 & 1.16319100 \\
\hline $\mathrm{H}$ & 0.34462400 & 1.53978600 & -0.24563800 \\
\hline $\mathrm{H}$ & -4.93794400 & 0.81318300 & -0.87702200 \\
\hline $\mathrm{C}$ & 3.74940500 & 0.78520600 & -0.25431100 \\
\hline
\end{tabular}




\begin{tabular}{|lrrr|}
\hline $\mathrm{H}$ & 4.59326900 & 1.09228000 & -0.88104600 \\
$\mathrm{H}$ & 3.99648700 & 1.08855000 & 0.77168800 \\
$\mathrm{H}$ & 0.00527800 & -0.66228300 & 1.94129800 \\
$\mathrm{H}$ & -0.14575900 & -2.06532000 & 0.89777200 \\
$\mathrm{C}$ & 1.06392200 & -0.48709700 & 0.06212200 \\
$\mathrm{H}$ & 0.87398000 & -0.76427900 & -0.98492000 \\
\hline
\end{tabular}

